\title{
Synthesis and Properties of 1,3-Disubstituted Ureas and Their Isosteric Analogs Containing Polycyclic Fragments: IV. ${ }^{1}$ 1-(Bicyclo[2.2.1]hept-5-en-2-yl)-3-(fluoro, chlorophenyl)ureas
}

\author{
D. A. Pitushkin ${ }^{a, b}$, V. V. Burmistrov ${ }^{a, b}$, and G. M. Butov ${ }^{a, b, *}$ \\ ${ }^{a}$ Volgograd State Technical University (VSTU), Volgograd, 400005 Russia \\ ${ }^{b}$ Volzhsky Polytechnic Institute, VSTU Branch, Volzhsky, 404121 Russia \\ *e-mail:butov@post.volpi.ru
}

Received April 13, 2020; revised April 23, 2020; accepted April 26, 2020

\begin{abstract}
The reaction of bicyclo[2.2.1]hept-5-en-2-yl isocyanate with fluoro- and chloro-substituted anilines was used to synthesize in a yield of $25-68 \%$ a series of 1,3-disubstituted ureas containing a lipophilic group in their structure. The synthesized ureas are promising as inhibitors of RNA virus replication and human soluble epoxide hydrolase.
\end{abstract}

Keywords: natural compounds, bicyclo[2.2.1]hept-5-ene, isocyanate, urea, halogen-containing anilines, soluble epoxide hydrolase, coronavirus, SARS-CoV

DOI: $10.1134 / \mathrm{S} 1070428020080023$

Isocyanates present interest as promising intermediates for the synthesis of biologically active substances. Ureas derived from isocyanates are versatile building blocks for heterocyclic synthesis and exhibit broad-spectrum biological activity [2]. For example, 2-(\{4'-[(phenylcarbamoyl)amino]-4-biphenylyl $\}$ carbonyl)cyclopentane1-carboxylic acid showed activity against rotavirus replication in cells and is considered as a potential antiviral agent [3]. A series of 1,3,3-trisubstituted ureas [ethyl 2-(4-R-1,4-diazepane-1-carboxamido)benzoates] was tested as inhibitors of RNA viruses, including SARS-CoV, and showed activity (the replication rate decreased to $7.97 \%$ of the control at a concentration of $250 \mu \mathrm{M}$ ), which makes these compounds potential antiviral agents against RNA viruses, including SARS-CoV, HIV-1, and other ARVI viruses [4]. Ureidothiophenes, the ureido group of which is linked the benzene ring, were found to act as powerful RNA polymerase inhibitors with antibacterial activity against Staphylococcus aureus and Staphylococcus epidermidis strains (MIC 1 and $0.25 \mu \mathrm{g} / \mathrm{mL}$, respectively) [5]. Over the past years 1,3-disubstituted ureas containing a highly lipophilic group have been actively explored as human soluble epoxide hydrolase [6].

1 For communication III, see [1].
Human soluble epoxide hydrolase (sEH), an enzyme involved in the metabolism of epoxy fatty acids to the corresponding vicinal diols via the addition of a water molecule, is a promising target in the therapy of hypertensive, inflammatory, and painful conditions [7]. The inhibition of this enzyme has a positive effect has a positive effect in the treatment of hypertension and kidney diseases [8].

Essential drawback of known sEH inhibitors are their fast metabolism under the action of P450 cytochrome and poor water solubility [9]. However, for high inhibitory activity toward $\mathrm{sEH}$, the urea molecule should necessarily contain a highly lipophilic fragment [10].

The above problem has been proposed to approach in different ways, including the synthesis of ureas comprising a natural fragment, specifically, bicyclo[2.2.1]hept-5-ene-2-yl (norbornene), found in the structures of compounds contained in sandalwood resin [11], pine resin [12], as well as birch bark and buds [13]. Wiseman et al. [14] showed in their study on the metabolism of sulfonylurea containing the norbornene fragment that the half-life of this compound in human plasma was $6 \mathrm{~h}$.

In this connection ureas containing a norbornene fragment can be considered as promising objects for further studies as metabolism-resistant, low cytotoxic, and highly 
biologically active inhibitors of RNA virus and human sEH inhibitors.

To synthesize inhibitors, we used the reaction of bicyclo[2.2.1] hept-5-ene-2-yl isocyanate (2) with halogensubstituted anilines.

Attempted synthesis of isocyanate $\mathbf{2}$ by the DielsAlder reaction of cyclopentadiaene with vinyl isocyanate gave only trace amounts of the product [15]. Phosgenation of 5-norbornene-2-amine or heating of 1-(bicyclo[2.2.1]hept-5-en-2-yl)urea in ethylene glycol in the presence of $\mathrm{KOH}$ gave isocyanate 2 in yields of up $70 \%$ [16]. Three procedures of the synthesis of isocyanate 2 by the Curtius reaction from bicyclo[2.2.1]hept-5-ene2-carboxylic acid (1) are known:

1) heating of chloride of acid $\mathbf{1}$ with sodium azide in anhydrous xylene (yield 47\%) [17];

2) reaction of acid $\mathbf{1}$ with ethyl chloroformate followed by addition of sodium azide at $-20^{\circ} \mathrm{C}$, isolation of the resulting acid 1 azide, and refluxing of the latter in ethyl acetate (no yield is specified) [18]; and

3) treatment of acid $\mathbf{1}$ with thionyl chloride followed by addition of the resulting acid $\mathbf{1}$ chloride to a boiling suspension of sodium azide in toluene (yield 83\%) [19]. The published procedures use toxic reagents, such as phosgene, ethyl chloroformate, and thionyl chloride.

The method of synthesis of isocyanate $\mathbf{2}$ (Scheme 1) proposed in the present work is free of most abovementioned drawbacks and, moreover, makes it possible to introduce the azido group in a carboxyl acid in one stage, avoiding acid chloride formation, and this strongly reduces reaction time.

Isocyanate 2 was prepared by the action of diphenoxyphosphinoyl azide (DPPA) on acid $\mathbf{1}$ in toluene under stirring in the presence of equimolar amount of triethylamine at $110^{\circ} \mathrm{C}$ for $2 \mathrm{~h}$.

Completion of the reaction was established by the cessation of nitrogen evolution from the reaction mixture.
The solvent was removed by vacuum distillation, and product $\mathbf{2}$ was separated from the triethylammonium salt that formed by extraction with diethyl ether.

To increase the yield of isocyanate 2 , we tried an alternative was of adding DPPA to the reaction mixture. Thus, instead of being added to the reaction mixture at $110^{\circ} \mathrm{C}$ over the course of $30 \mathrm{~min}$, DPPA was added in one portion at room temperature. It was found that the new method of addition of DPPA only slightly affected the yield of isocyanate 2 but simplified the procedure considerably.

Isocyanate $\mathbf{2}$ was reacted with haloanilines $\mathbf{3 a}-\mathbf{3 n}$ to obtain ureas $4 \mathbf{a}-\mathbf{4 n}$ (Scheme 2). Choosing haloanilines we focused on the possibility to obtain a correlation between the activity of the ureas on the positions of the $\mathrm{F}$ and/or $\mathrm{Cl}$ atoms and the number of halogen atoms.

It should be noted that the procedure for synthesis and isolation of adamantyl-substituted ureas, with DMF as a solvent, proved to be unsuitable in our case. Therefore, the reaction was performed in anhydrous diethyl ether in the presence of triethylamine at at room temperature for $12 \mathrm{~h}$.

After the reaction in DMF, ureas were isolated by adding $1 \mathrm{~N} \mathrm{HCl}$ to the reaction mixture, and, therewith, the solvent was not removed.

As a result, the ureas precipitated and then filtered off and washed with water [7]. In the present work, attempted isolation of $\mathbf{4 f}, \mathbf{4 h}$, and $\mathbf{4 i}$ from the reaction mixture by such procedure failed. Increasing the volume of the $\mathrm{HCl}$ solution added to the reaction mixture, increasing the concentration of the $\mathrm{HCl}$ solution, and removing DMF did not have a positive effect. Treatment of the reaction mixture resulted in the formation of a liquid phase immiscible with water, from which the target compounds were impossible to isolate in sufficient amounts. Presumably, this effect takes place due to the fact that the resulting ureas are better soluble in DMF than their adamantyl-containing analogs. When the

Scheme 1.

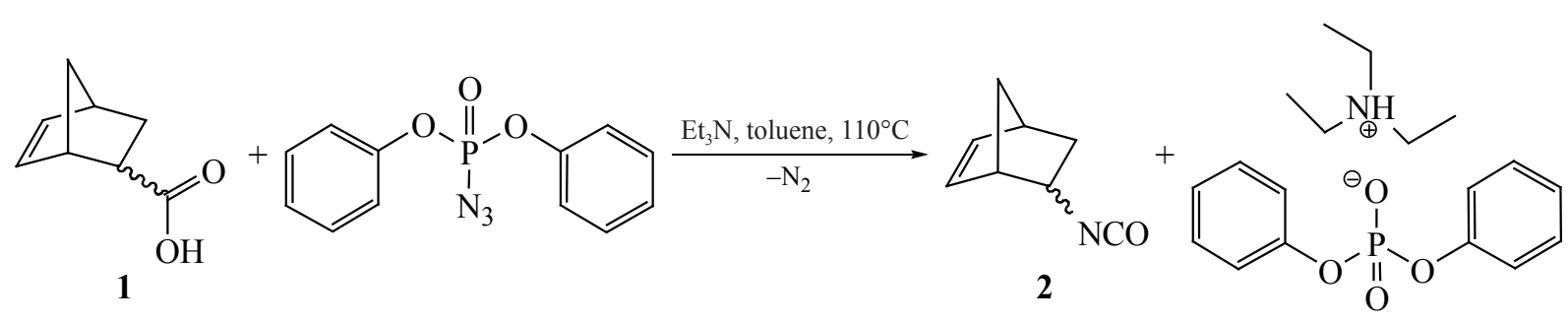

RUSSIAN JOURNAL OF ORGANIC CHEMISTRY Vol. 56 No. 82020 
Scheme 2.

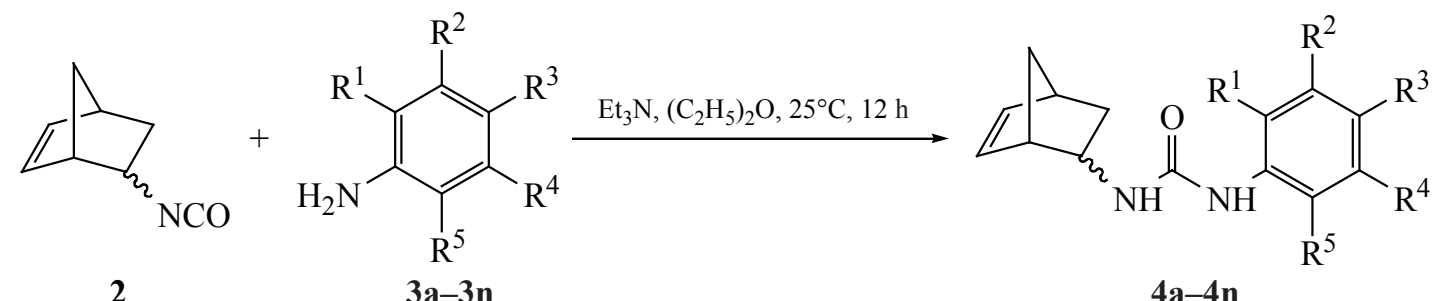

2

$$
\begin{array}{r}
\mathrm{R}^{2}=\mathrm{F}, \mathrm{R}^{1}=\mathrm{R}^{3}=\mathrm{R}^{4}=\mathrm{R}^{5}=\mathrm{H}(\mathbf{4} \mathbf{a}) ; \mathrm{R}^{3}=\mathrm{F}, \mathrm{R}^{1}=\mathrm{R}^{2}=\mathrm{R}^{4}=\mathrm{R}^{5}=\mathrm{H}(\mathbf{4} \mathbf{b}) ; \mathrm{R}^{2}=\mathrm{Cl}, \mathrm{R}^{1}=\mathrm{R}^{3}=\mathrm{R}^{4}=\mathrm{R}^{5}=\mathrm{H}(\mathbf{4} \mathbf{c}) ; \\
\mathrm{R}^{1}=\mathrm{R}^{3}=\mathrm{F}, \mathrm{R}^{2}=\mathrm{R}^{4}=\mathrm{R}^{5}=\mathrm{H}(\mathbf{4 d}) ; \mathrm{R}^{1}=\mathrm{R}^{5}=\mathrm{F}, \mathrm{R}^{2}=\mathrm{R}^{3}=\mathrm{R}^{4}=\mathrm{H}(\mathbf{4 e}) ; \mathrm{R}^{2}=\mathrm{R}^{3}=\mathrm{F}, \mathrm{R}^{1}=\mathrm{R}^{4}=\mathrm{R}^{5}=\mathrm{H}(\mathbf{4 f}) ; \\
\mathrm{R}^{1}=\mathrm{R}^{4}=\mathrm{F}, \mathrm{R}^{2}=\mathrm{R}^{3}=\mathrm{R}^{5}=\mathrm{H}(\mathbf{4} \mathbf{g}) ; \mathrm{R}^{2}=\mathrm{R}^{4}=\mathrm{F}, \mathrm{R}^{1}=\mathrm{R}^{3}=\mathrm{R}^{5}=\mathrm{H}(\mathbf{4 h}) ; \mathrm{R}^{1}=\mathrm{Cl}_{1}, \mathrm{R}^{3}=\mathrm{F}, \mathrm{R}^{2}=\mathrm{R}^{4}=\mathrm{R}^{5}=\mathrm{H}(\mathbf{4} \mathbf{i}) ; \\
\mathrm{R}^{2}=\mathrm{Cl}, \mathrm{R}^{3}=\mathrm{F}, \mathrm{R}^{1}=\mathrm{R}^{4}=\mathrm{R}^{5}=\mathrm{H}(\mathbf{4 j}) ; \mathrm{R}^{1}=\mathrm{F}, \mathrm{R}^{2}=\mathrm{Cl}, \mathrm{R}^{3}=\mathrm{R}^{4}=\mathrm{R}^{5}=\mathrm{H}(\mathbf{4 k}) ; \\
\mathrm{R}^{2}=\mathrm{F}, \mathrm{R}^{3}=\mathrm{Cl}, \mathrm{R}^{1}=\mathrm{R}^{4}=\mathrm{R}^{5}=\mathrm{H}(\mathbf{4} \mathbf{l}) ; \mathrm{R}^{1}=\mathrm{F}, \mathrm{R}^{3}=\mathrm{Cl}, \mathrm{R}^{2}=\mathrm{R}^{4}=\mathrm{R}^{5}=\mathrm{H}(\mathbf{4} \mathbf{m}) ; \\
\mathrm{R}^{2}=\mathrm{Cl}, \mathrm{R}^{5}=\mathrm{F}, \mathrm{R}^{1}=\mathrm{R}^{3}=\mathrm{R}^{4}=\mathrm{H}(\mathbf{4 n})
\end{array}
$$

reaction was performed in diethyl ether, the target urea precipitated after removal of the solvent and addition of $1 \mathrm{~N} \mathrm{HCl}$ and was filtered off and washed with distilled water.

The properties of the synthesized 1,3-disubstituted ureas $\mathbf{4 a}-\mathbf{4 n}$ are listed in Table 1.

The lipophilicity coefficients of compounds $\mathbf{4 a}-\mathbf{4 n}$ span the range 3.10-3.73, which satisfies the Lipinski's rule [21] with a large margin and is, on average, 1.5 units lower than that of related adamantyl-containing analogs.

Decreasing melting point positively affects the properties of ureas, facilitating the preparation of dosage forms [22]. The melting points of ureas $\mathbf{4 a - 4 n}$ depend on the number and positions of substituents in the aromatic ring, as well as on the nature of the halogen atom. The replacement of fluorine in the 3-position by chlorine (compounds $4 \mathbf{a}$ and $\mathbf{4 c}$ ) decreases the melting point by $48^{\circ} \mathrm{C}$ (181 and $133^{\circ} \mathrm{C}$, respectively). Two fluorine substituents in the aromatic ring decrease the melting point of ureas $\mathbf{4 d}-\mathbf{4 h}$ by $11-60^{\circ} \mathrm{C}$ compared to compound $\mathbf{4 b}$. Fluorine in the 4-position (4b) decreases the melting point by $14^{\circ} \mathrm{C}$ compared to compound $4 \mathrm{a}$, where the $3-\mathrm{F}$ substituent is present. One more fluorine substituent in the 2-position along with the 4-F substituent (compound 4d) decreases the melting point by $47^{\circ} \mathrm{C}$ compared to compound $\mathbf{4 b}$, whereas the introduction of the fluorine substituent in the 3-position (compound $\mathbf{4 f}$ ) decreases the melting point by $24^{\circ} \mathrm{C}$.

The replacement of the fluorine substituent in the 3-position of the aromatic ring by chlorine, provided the molecule contains one more fluorine substituent (compounds $4 \mathbf{j}, \mathbf{4 k}$, and $\mathbf{4 n}$ ), the melting point increases by
7-20 ${ }^{\circ} \mathrm{C}$ compared to compound $\mathbf{4 a}$, whereas the same exchange in the 4-position (compounds $\mathbf{4 l}$ and $\mathbf{4 m}$ ), by contrast, decreases the melting point by $20-73^{\circ} \mathrm{C}$ compared to compound $\mathbf{4 b}$. The introduction of the chlorine atom to the 2-position of the aromatic ring (compound 4i) decreases the melting point by $32^{\circ} \mathrm{C}$ compounds to monofluorinated compound $\mathbf{4 b}$.

Comparing the melting point of compound $\mathbf{4 c}$ with those of its adamantane analogs we can note that the bicyclo[2.2.1] hept-5-enyl fragment decreases the melting point by $27^{\circ} \mathrm{C}$ compared to the adamantyl [20] and by $56^{\circ} \mathrm{C}$ compared to the 4-oxoadamantyl fragments [23].

In general, two fluorine substituents in the aromatic ring favor a lower melting point. When two different substituents (fluorine and chlorine) are present in the aromatic ring, chlorine is preferable to be present in the 2- or 4-position, while the position of the fluorine substituent is not important. By contrast, the replacement of chlorine by fluorine in the 3-position of the aromatic ring increases the melting point.

Since the starting isocyanate 2 consists of the endoand exo-isomers, the ureas derived from this compound, too, are mixtures of the two isomers. The ${ }^{1} \mathrm{H}$ NMR signals of the endo- and exo-isomers have quite different chemical shifts. The proton signal of the $\mathrm{NH}$ group remote from the bicyclic fragment appears at 5.28-6.38 ppm for the exo-isomer and at 6.35-7.12 ppm for the endo-isomer, and the gap between the signals of the two different isomers is, on average, $0.7 \mathrm{ppm}$. The proton signal of the $\mathrm{NH}$ group proximate to the aromatic ring appears at $7.69-8.96 \mathrm{ppm}$ for the exo isomer and at $7.62-8.88 \mathrm{ppm}$ for the endo-isomer; however, the gap between the signals 
Table 1. Lipophilicity coefficients, melting points, and yields of compounds $4 \mathbf{a}-\mathbf{4 n}$ and their analogs

\begin{tabular}{|c|c|c|c|c|c|c|c|c|c|}
\hline Compound no. & $\mathrm{R}^{1}$ & $\mathrm{R}^{2}$ & $\mathrm{R}^{3}$ & $\mathrm{R}^{4}$ & $\mathrm{R}^{5}$ & $M_{\mathrm{r}}$ & $\mathrm{mp},{ }^{\circ} \mathrm{C}$ & $\log P^{\mathrm{a}}$ & Yield, \% \\
\hline $4 a$ & $\mathrm{H}$ & $\mathrm{F}$ & $\mathrm{H}$ & $\mathrm{H}$ & $\mathrm{H}$ & 246 & $181-182$ & 3.10 & 50 \\
\hline $4 b$ & $\mathrm{H}$ & $\mathrm{H}$ & $\mathrm{F}$ & $\mathrm{H}$ & $\mathrm{H}$ & 246 & $167-168$ & 3.12 & 60 \\
\hline $4 c$ & $\mathrm{H}$ & $\mathrm{Cl}$ & $\mathrm{H}$ & $\mathrm{H}$ & $\mathrm{H}$ & 262 & $133-134$ & 3.61 & 47 \\
\hline $4 d$ & $\mathrm{~F}$ & $\mathrm{H}$ & $\mathrm{F}$ & $\mathrm{H}$ & $\mathrm{H}$ & 264 & $121-122$ & 3.21 & 39 \\
\hline $4 e$ & $\mathrm{~F}$ & $\mathrm{H}$ & $\mathrm{H}$ & $\mathrm{H}$ & $\mathrm{F}$ & 264 & $170-171$ & 3.19 & 26 \\
\hline $4 f$ & $\mathrm{H}$ & $\mathrm{F}$ & $\mathrm{F}$ & $\mathrm{H}$ & $\mathrm{H}$ & 264 & $143-144$ & 3.21 & 61 \\
\hline $4 g$ & $\mathrm{~F}$ & $\mathrm{H}$ & $\mathrm{H}$ & $\mathrm{F}$ & $\mathrm{H}$ & 264 & $146-147$ & 3.21 & 47 \\
\hline $4 h$ & $\mathrm{H}$ & $\mathrm{F}$ & $\mathrm{H}$ & $\mathrm{F}$ & $\mathrm{H}$ & 264 & $143-144$ & 3.21 & 29 \\
\hline $4 i$ & $\mathrm{Cl}$ & $\mathrm{H}$ & $\mathrm{F}$ & $\mathrm{H}$ & $\mathrm{H}$ & 280 & $135-136$ & 3.71 & 35 \\
\hline $4 j$ & $\mathrm{H}$ & $\mathrm{Cl}$ & $\mathrm{F}$ & $\mathrm{H}$ & $\mathrm{H}$ & 280 & $188-189$ & 3.73 & 68 \\
\hline $4 k$ & $\mathrm{~F}$ & $\mathrm{Cl}$ & $\mathrm{H}$ & $\mathrm{H}$ & $\mathrm{H}$ & 280 & $201-202$ & 3.70 & 25 \\
\hline 41 & $\mathrm{H}$ & $\mathrm{F}$ & $\mathrm{Cl}$ & $\mathrm{H}$ & $\mathrm{H}$ & 280 & $93-94$ & 3.73 & 65 \\
\hline $4 m$ & $\mathrm{~F}$ & $\mathrm{H}$ & $\mathrm{Cl}$ & $\mathrm{H}$ & $\mathrm{H}$ & 280 & $147-148$ & 3.73 & 41 \\
\hline $4 n$ & $\mathrm{H}$ & $\mathrm{Cl}$ & $\mathrm{H}$ & $\mathrm{H}$ & $\mathrm{F}$ & 280 & $195-196$ & 3.73 & 27 \\
\hline & & & & & & 304 & $160-161[20]$ & 5.02 & 73 [20] \\
\hline
\end{tabular}

${ }^{a}$ Calculated by the Molinspiration software (http://www.molinspiration.com).

of the two different isomers is naturally smaller, specifically, $0.08 \mathrm{ppm}$. The ${ }^{19} \mathrm{~F}$ NMR spectra of compounds $\mathbf{4 d}, \mathbf{4 g}, \mathbf{4 h}$, and $\mathbf{4 i}$, too, contain doubled signals corresponding to the exo- and endo-isomers. The gap between the fluorine signals of the exo- and endo-isomers is $0.31-2.13 \mathrm{ppm}$.

An interesting feature of the synthesized ureas $\mathbf{4 a}-\mathbf{4 n}$, which differentiates them from the adamantyl-containing analogs, is their stability under the conditions of the GCMS experiment. We earlier showed that adamantylcontaining 1,3-disubstituted ureas undergo decomposition in a $\mathrm{GC}$ injector at $250^{\circ} \mathrm{C}$ [24]. The GCMS chromatograms of unsymmetrical adamantyl-containing ureas showed 4 peaks, which, according to the mass spectra, correspond to amines and isocyanates formed by the dissociation of the urea moiety along two possible routes (Scheme 3). The GCMS chromatograms showed no peaks, the mass spectra of which would contain molecular or fragment ions assignable to adamantyl-substituted ureas. To detect such compounds one should make use either of direct-inlet mass spectrometry or HPLC.

However, in the case of ureas containing the bicyclo[2.2.1] hept-5-en-2-yl fragment (except for $\mathbf{4 c}, \mathbf{4 j}$, and 4l), the GCMS chromatograms contain two peaks assignable to the endo- and exo-isomers of the corresponding products. Table 2 lists the retention times of ureas $\mathbf{4 a}-\mathbf{4 n}$ and characteristic fragment ions in the EI mass spectra. 


\section{Scheme 3.}

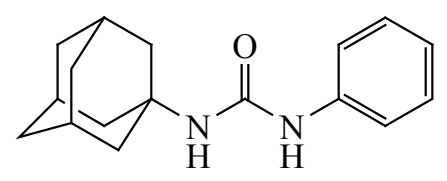

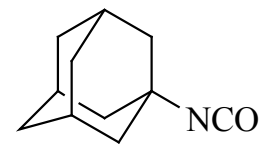

$m / z 177$

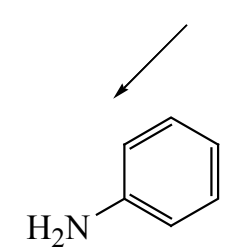

$m / z 93$
Thus, a series of 1-(bicyclo[2.2.1] hept-5-en-2-yl)-3-Rdisubstituted ureas, where $\mathrm{R}$ is a halogenated aromatic group, was prepared in mild conditions. The lipophilicity coefficients of the synthesized compounds are, on average, lower by 1.5 units, compared to the respective values of their adamantyl analog [1]. Thus, the replacement of the adamantyl fragment in the inhibitor molecule by norbornene decreases the lipophilicity of 1,3-disubstituted ureas. The synthesized compounds will be tested as potential inhibitors of RNA virus and human sEH inhibitors.

\section{EXPERIMENTAL}

3-Chloroaniline (99\%, CAS 108-42-9), 3-chloro4-fluoroaniline (98\%, CAS 367-21-5), triethylamine (BioUltra $\geq 99.5 \%$, CAS 121-44-8), and DMF (anhydrous, 99.8\%, CAS 68-12-2) from Sigma-Aldrich; 4-fluoroaniline (99\%, CAS 371-40-4), 2,4-difluoroaniine (99\%, CAS 367-25-9), 2,6-difluoroaniline (98\%, CAS 5509-65-9), 3,4-difluoroaniline (98\%, CAS 5509-65-9), 3,4-difluoroaniline (98+\%, CAS 3863-11-4), 2-chloro-4-fluoroaniline (97\%, CAS 2106-02-7), 4-chloro-2-fluoroaniline (98\%, CAS 57946-56-2), 5-chloro-2-fluoroaniline (97\%, CAS 2106-05-0), and 2,5-difluoroaniline (98\%, CAS 367-30-6) from Alfa Aesar; 3-fluoroaniline (98\%, CAS 372-19-0) from abcr; 3-chloro-2-fluoroaniline (98\%, CAS 2106-04-9) and 4-chloro-3-fluoroaniline (98\%, CAS 367-22-6) from J\&K; and 3,5-difluoroaniline (98\%, CAS 372-39-4) from Fluorochem were used as received.

The structure of the synthesized compounds was confirmed by ${ }^{1} \mathrm{H}$ and ${ }^{13} \mathrm{C}$ NMR spectroscopy, GCMS, and elemental analysis. The mass spectra were obtained on an Agilent GC 5975/MSD 7820 system; chromatographic separation was performed on an HP-5MS quartz capillary column (length $30 \mathrm{~m}$, diameter $0.25 \mathrm{~mm}$, film thickness $0.5 \mu \mathrm{m})$, carrier gas helium, temperature ramp from 80 to $280^{\circ} \mathrm{C}$; injector temperature $250^{\circ} \mathrm{C}$, electron ionization.

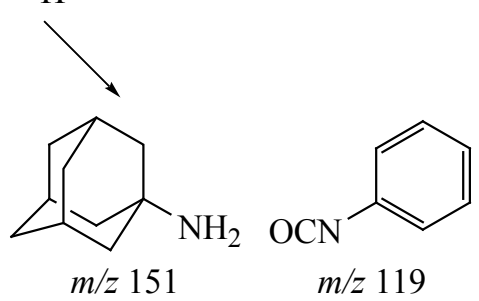

The ${ }^{1} \mathrm{H},{ }^{13} \mathrm{C}$, and ${ }^{19} \mathrm{~F}$ NMR spectra were obtained on a Bruker DRX500 spectrometer at 500.13, 125.76, and $470.59 \mathrm{MHz}$, respectively, in DMSO- $d_{6}$; the ${ }^{1} \mathrm{H}$ chemical shifts were measured against internal TMS. The elemental analyses were obtained on a Perkin-Elmer Series II 2400 analyzer.

Bicyclo[2.2.1]hept-5-en-2-yl isocyanate (2). Triethylamine, $5.24 \mathrm{~mL}(36.23 \mathrm{mmol})$, and $10.46 \mathrm{~g}$ (38.03 mmol) of diphenoxyphosphinoyl azide were added to a solution of $5.0 \mathrm{~g}(36.23 \mathrm{mmol})$ of bicyclo[2.2.1] hept-5-ene-2-carboxylic acid in $50 \mathrm{~mL}$ of toluene. The reaction mixture was slowly heated with stirring until boiling and then refluxed for $2 \mathrm{~h}$. The reaction completion was established, when nitrogen no longer evolved from the reaction mixture. After cooling to room temperature, the solvent was removed under reduced pressure to leave a yellow oily residue, from which the product was extracted with diethyl ether $(2 \times 15 \mathrm{~mL})$. The combined extract was evaporated in a vacuum to obtain a transparent oily liquid. Yield $3.45 \mathrm{~g}$ (70\%). ${ }^{1} \mathrm{H}$ NMR spectrum (DMSO$\left.d_{6}\right), \delta$, ppm: 0.52 d.t $\left(1 \mathrm{H}\right.$, endo- $\mathrm{C} \underline{\mathrm{H}}-\mathrm{CH}_{2}-\mathrm{CH}-\mathrm{NCO}, J_{1}$ $\left.11.7, J_{2} 3.2 \mathrm{~Hz}\right), 1.07$ d.t $\left(1 \mathrm{H}\right.$, exo-C $\underline{\mathrm{H}}-\mathrm{CH}_{2}-\mathrm{CH}-\mathrm{NCO}$, $\left.J_{1} 11.9, J_{2} 3.4 \mathrm{~Hz}\right), 1.28 \mathrm{q}\left(2 \mathrm{H}\right.$, endo- $\left.\mathrm{CH}_{2}, J 8.3 \mathrm{~Hz}\right)$, $1.40 \mathrm{q}\left(2 \mathrm{H}\right.$, exo- $\left.\mathrm{CH}_{2}, J 8.4 \mathrm{~Hz}\right), 1.78-1.84 \mathrm{~m}(1 \mathrm{H}$, exoC $\underline{H}-\mathrm{CH}-\mathrm{NCO}), 2.02-2.08 \mathrm{~m}(1 \mathrm{H}$, exo-C $\underline{\mathrm{H}}-\mathrm{CH}-\mathrm{NCO})$, $2.57 \mathrm{~s}\left(1 \mathrm{H}\right.$, endo- $\left.\mathrm{CH}-\mathrm{C}_{2}-\mathrm{CH}-\mathrm{NCO}\right), 2.75 \mathrm{~s}(1 \mathrm{H}$, exo$\left.\mathrm{CH}-\mathrm{CH}_{2}-\mathrm{CH}-\mathrm{NCO}\right), 2.79 \mathrm{~s}\left(1 \mathrm{H}\right.$, endo- $\mathrm{CH}-\mathrm{C}_{2}-\mathrm{CH}-$ $\mathrm{NCO}), 2.84 \mathrm{~s}\left(1 \mathrm{H}\right.$, exo-CH- $\left.\underline{\mathrm{H}}_{2}-\mathrm{CH}-\mathrm{NCO}\right), 3.39-3.44 \mathrm{~m}$ (1H, endo- $\mathrm{C} \underline{\mathrm{H}}-\mathrm{NCO}), 4.12-4.18 \mathrm{~m}(1 \mathrm{H}$, exo- $\mathrm{C} \underline{\mathrm{H}}-\mathrm{NCO})$, $5.98 \mathrm{q}(1 \mathrm{H}$, endo- $\mathrm{C} \underline{\mathrm{H}}=\mathrm{CH}-\mathrm{CH}-\mathrm{CH}-\mathrm{NCO}, J 2.8 \mathrm{~Hz})$, $6.04 \mathrm{q}(1 \mathrm{H}$, exo-C $\underline{\mathrm{H}}=\mathrm{CH}-\mathrm{CH}-\mathrm{CH}-\mathrm{NCO}, J 2.8 \mathrm{~Hz}), 6.11 \mathrm{q}$ $(1 \mathrm{H}$, endo- $\mathrm{CH}=\mathrm{C} \underline{\mathrm{H}}-\mathrm{CH}-\mathrm{CH}-\mathrm{NCO}, J 2.8 \mathrm{~Hz}) 6.32 \mathrm{q}(1 \mathrm{H}$, exo- $\mathrm{CH}=\mathrm{C} \underline{\mathrm{H}}-\mathrm{CH}-\mathrm{CH}-\mathrm{NCO}, J 2.9 \mathrm{~Hz}) .{ }^{13} \mathrm{C}$ NMR spectrum (DMSO- $\left.d_{6}\right), \delta$, ppm: 36.71 (endo- $\underline{\mathrm{H}}_{2}-\mathrm{CH}-\mathrm{NCO}$ ), 37.18 (exo- $\mathrm{CH}_{2}-\mathrm{CH}-\mathrm{NCO}$ ), 41.13 (endo- $\mathrm{CH}-\mathrm{CH}_{2}-\mathrm{CH}-$ $\mathrm{NCO}$ ), 42.74 (exo- $\mathrm{CH}-\mathrm{CH}_{2}-\mathrm{CH}-\mathrm{NCO}$ ), 45.93 (endo- 
Table 2. Gas chromatography-mass spectrometry data for ureas $\mathbf{4 a - 4 n}$

\begin{tabular}{|c|c|c|c|c|c|c|c|}
\hline Compound no. & $\mathrm{R}^{1}$ & $\mathrm{R}^{2}$ & $\mathrm{R}^{3}$ & $\mathrm{R}^{4}$ & $\mathrm{R}^{5}$ & $\begin{array}{l}\text { Retention time } \\
\text { endo/exo, min }\end{array}$ & Characteristic ions \\
\hline $4 \mathbf{a}$ & $\mathrm{H}$ & $\mathrm{F}$ & $\mathrm{H}$ & $\mathrm{H}$ & $\mathrm{H}$ & $18.320 / 18.596$ & $\begin{array}{c}246[M]^{+}, 180\left[\mathrm{~F}-\mathrm{Ph}-\mathrm{NH}-\mathrm{C}(\mathrm{O})-\mathrm{NH}-\mathrm{CH}=\mathrm{CH}_{2}\right]^{+} \\
137[\mathrm{~F}-\mathrm{Ph}-\mathrm{NCO}], 111\left[\mathrm{~F}-\mathrm{Ph}-\mathrm{NH}_{2}\right]^{+}\end{array}$ \\
\hline $4 b$ & $\mathrm{H}$ & $\mathrm{H}$ & $\mathrm{F}$ & $\mathrm{H}$ & $\mathrm{H}$ & $18.116 / 18.387$ & $\begin{array}{c}246[M]^{+}, 180\left[\mathrm{~F}-\mathrm{Ph}-\mathrm{NH}-\mathrm{C}(\mathrm{O})-\mathrm{NH}-\mathrm{CH}=\mathrm{CH}_{2}\right]^{+} \\
137[\mathrm{~F}-\mathrm{Ph}-\mathrm{NCO}], 111\left[\mathrm{~F}-\mathrm{Ph}-\mathrm{NH}_{2}\right]^{+}\end{array}$ \\
\hline $4 d$ & $\mathrm{~F}$ & $\mathrm{H}$ & $\mathrm{F}$ & $\mathrm{H}$ & $\mathrm{H}$ & $17.154 / 17.433$ & $\begin{array}{c}264[M]^{+}, 198\left[\mathrm{~F}_{2}-\mathrm{Ph}-\mathrm{NH}-\mathrm{C}(\mathrm{O})-\mathrm{NH}-\mathrm{CH}=\mathrm{CH}_{2}\right]^{+} \\
155\left[\mathrm{~F}_{2}-\mathrm{Ph}-\mathrm{NCO}\right], 129\left[\mathrm{~F}_{2}-\mathrm{Ph}-\mathrm{NH}_{2}\right]^{+}\end{array}$ \\
\hline $4 e$ & $\mathrm{~F}$ & $\mathrm{H}$ & $\mathrm{H}$ & $\mathrm{H}$ & $\mathrm{F}$ & $17.094 / 17.321$ & $\begin{array}{c}264[M]^{+}, 198\left[\mathrm{~F}_{2}-\mathrm{Ph}-\mathrm{NH}-\mathrm{C}(\mathrm{O})-\mathrm{NH}-\mathrm{CH}=\mathrm{CH}_{2}\right]^{+} \\
155\left[\mathrm{~F}_{2}-\mathrm{Ph}-\mathrm{NCO}\right], 129\left[\mathrm{~F}_{2}-\mathrm{Ph}-\mathrm{NH}_{2}\right]^{+}\end{array}$ \\
\hline $4 f$ & $\mathrm{H}$ & $\mathrm{F}$ & $\mathrm{F}$ & $\mathrm{H}$ & $\mathrm{H}$ & $18.375 / 18.673$ & $\begin{array}{c}264[M]^{+}, 198\left[\mathrm{~F}_{2}-\mathrm{Ph}-\mathrm{NH}-\mathrm{C}(\mathrm{O})-\mathrm{NH}-\mathrm{CH}=\mathrm{CH}_{2}\right]^{+} \\
155\left[\mathrm{~F}_{2}-\mathrm{Ph}-\mathrm{NCO}\right], 129\left[\mathrm{~F}_{2}-\mathrm{Ph}-\mathrm{NH}_{2}\right]^{+}\end{array}$ \\
\hline $4 \mathrm{~g}$ & $\mathrm{~F}$ & $\mathrm{H}$ & $\mathrm{H}$ & $\mathrm{F}$ & $\mathrm{H}$ & $17.524 / 17.833$ & $\begin{array}{c}264[M]^{+}, 198\left[\mathrm{~F}_{2}-\mathrm{Ph}-\mathrm{NH}-\mathrm{C}(\mathrm{O})-\mathrm{NH}-\mathrm{CH}=\mathrm{CH}_{2}\right]^{+} \\
155\left[\mathrm{~F}_{2}-\mathrm{Ph}-\mathrm{NCO}\right], 129\left[\mathrm{~F}_{2}-\mathrm{Ph}-\mathrm{NH}_{2}\right]^{+}\end{array}$ \\
\hline $4 h$ & $\mathrm{H}$ & $\mathrm{F}$ & $\mathrm{H}$ & $\mathrm{F}$ & $\mathrm{H}$ & $18.148 / 18.445$ & $\begin{array}{c}264\left[\mathrm{M}^{+}, 198\left[\mathrm{~F}_{2}-\mathrm{Ph}-\mathrm{NH}-\mathrm{C}(\mathrm{O})-\mathrm{NH}-\mathrm{CH}=\mathrm{CH}_{2}\right]^{+}\right. \\
155\left[\mathrm{~F}_{2}-\mathrm{Ph}-\mathrm{NCO}\right], 129\left[\mathrm{~F}_{2}-\mathrm{Ph}-\mathrm{NH}_{2}\right]^{+}\end{array}$ \\
\hline $4 i$ & $\mathrm{Cl}$ & $\mathrm{H}$ & $\mathrm{F}$ & $\mathrm{H}$ & $\mathrm{H}$ & $18.608 / 18.871$ & $\begin{array}{c}280\left[\mathrm{M}^{+}, 214\left[\mathrm{FCl}-\mathrm{Ph}-\mathrm{NH}-\mathrm{C}(\mathrm{O})-\mathrm{NH}-\mathrm{CH}=\mathrm{CH}_{2}\right]^{+},\right. \\
171[\mathrm{FCl}-\mathrm{Ph}-\mathrm{NCO}], 145\left[\mathrm{FCl}-\mathrm{Ph}-\mathrm{NH}_{2}\right]^{+}\end{array}$ \\
\hline $4 k$ & $\mathrm{~F}$ & $\mathrm{Cl}$ & $\mathrm{H}$ & $\mathrm{H}$ & $\mathrm{H}$ & $19.554 / 19.815$ & $\begin{array}{c}280\left[\mathrm{M}^{+}, 214\left[\mathrm{FCl}-\mathrm{Ph}-\mathrm{NH}-\mathrm{C}(\mathrm{O})-\mathrm{NH}-\mathrm{CH}=\mathrm{CH}_{2}\right]^{+},\right. \\
171[\mathrm{FCl}-\mathrm{Ph}-\mathrm{NCO}], 145\left[\mathrm{FCl}-\mathrm{Ph}-\mathrm{NH}_{2}\right]^{+}\end{array}$ \\
\hline $4 m$ & $\mathrm{~F}$ & $\mathrm{H}$ & $\mathrm{Cl}$ & $\mathrm{H}$ & $\mathrm{H}$ & $19.255 / 19.526$ & $\begin{array}{c}280\left[\mathrm{M}^{+}, 214\left[\mathrm{FCl}-\mathrm{Ph}-\mathrm{NH}-\mathrm{C}(\mathrm{O})-\mathrm{NH}-\mathrm{CH}=\mathrm{CH}_{2}\right]^{+},\right. \\
171[\mathrm{FCl}-\mathrm{Ph}-\mathrm{NCO}], 145\left[\mathrm{FCl}-\mathrm{Ph}-\mathrm{NH}_{2}\right]^{+}\end{array}$ \\
\hline $4 n$ & $\mathrm{H}$ & $\mathrm{Cl}$ & $\mathrm{H}$ & $\mathrm{H}$ & $\mathrm{F}$ & $19.424 / 19.705$ & $\begin{array}{c}280\left[\mathrm{M}^{+}, 214\left[\mathrm{FCl}-\mathrm{Ph}-\mathrm{NH}-\mathrm{C}(\mathrm{O})-\mathrm{NH}-\mathrm{CH}=\mathrm{CH}_{2}\right]^{+},\right. \\
171[\mathrm{FCl}-\mathrm{Ph}-\mathrm{NCO}], 145\left[\mathrm{FCl}-\mathrm{Ph}-\mathrm{NH}_{2}\right]^{+}\end{array}$ \\
\hline
\end{tabular}

$\underline{\mathrm{C}} \mathrm{H}-\mathrm{NCO}), 47.80$ (exo- $\underline{\mathrm{CH}}-\mathrm{NCO}), 48.09$ (endo- $\mathrm{CH}_{2}$ bridge), 50.18 (exo- $\mathrm{CH}_{2}$ bridge), 52.95 (endo- $\mathrm{CH}-\mathrm{CH}-$ $\mathrm{NCO}$ ), 53.38 (exo- $\mathrm{CH}-\mathrm{CH}-\mathrm{NCO}), 131.43$ (endo-NCO), 133.32 (exo-NCO), 139.66 (2C, endo- $\underline{\mathrm{C}} \mathrm{H}=\underline{\mathrm{CH}}), 139.89$ $(2 \mathrm{C}$, exo- $\underline{\mathrm{CH}}=\underline{\mathrm{CH}})$. Mass spectrum, $m / z\left(I_{\text {rel }}, \%\right): 135(2.0)$ $[M]^{+}, 120$ (4.0) $\left[M-\mathrm{CH}_{3}\right]^{+}, 107$ (12.0) $\left[M-2 \mathrm{CH}_{2}\right]^{+}, 93$ (10.0) $\left[M-\mathrm{NCO}^{+}\right], 66$ (100) $\left[M-\mathrm{CH}_{2}-\mathrm{CH}-\mathrm{NCO}\right]^{+}$. Found, \%: C 71.12; H 6.67; N 10.39. $\mathrm{C}_{8} \mathrm{H}_{9} \mathrm{NO}$. Calculated, \%: C 71.09; H 6.71; N 10.36. M 135.16.

1-(Bicyclo[2.2.1]hept-5-en-2-yl)-3-(3-fluorophenyl)urea (4a). To a solution of $0.2 \mathrm{~g}(1.48 \mathrm{mmol})$ of bicyclo[2.2.1]hept-5-en-2-yl isocyanate (2) in $5 \mathrm{~mL}$ of anhydrous diethyl ether, $0.164 \mathrm{~g}(1.48 \mathrm{mmol})$ of 3-fluo- roaniline (3a) and $0.2 \mathrm{~mL}(1.48 \mathrm{mmol})$ of triethylamine were added. The reaction mixture was stirred at room temperature for $12 \mathrm{~h}$, after which the solvent was removed by vacuum distillation. The residue was then poured with $5 \mathrm{~mL}$ of $1 \mathrm{~N} \mathrm{HCl}$, the mixture was stirred for an additional $30 \mathrm{~min}$, and the precipitate that formed was filtered off and washed with water. Yield $0.18 \mathrm{~g} \mathrm{(50 \% ),}$ mp $181-182^{\circ} \mathrm{C} .{ }^{1} \mathrm{H}$ NMR spectrum (DMSO- $\left.d_{6}\right), \delta$, ppm: 0.62 d.t $\left(1 \mathrm{H}\right.$, endo- $\left.\underline{\mathrm{CH}}-\mathrm{CH}_{2}-\mathrm{CH}-\mathrm{NH}, J_{1} 11.8, J_{2} 3.2 \mathrm{~Hz}\right)$, 1.06 d.t $\left(1 \mathrm{H}\right.$, exo- $\left.\mathrm{CH}-\mathrm{CH}_{2}-\mathrm{CH}-\mathrm{NH}, J_{1} 12.0, J_{2} 3.4 \mathrm{~Hz}\right)$, $1.34 \mathrm{q}\left(2 \mathrm{H}\right.$, endo- $\left.\mathrm{CH}_{2}, J 8.4 \mathrm{~Hz}\right), 1.57 \mathrm{q}\left(2 \mathrm{H}\right.$, exo- $\mathrm{CH}_{2}$, $J 8.4 \mathrm{~Hz}), 2.12$ d.d.d $\left(1 \mathrm{H}, \mathrm{C} \underline{\mathrm{H}}-\mathrm{CH}-\mathrm{NH}, J_{1} 12.2, J_{2} 8.8\right.$, $\left.J_{3} 3.8 \mathrm{~Hz}\right), 2.68 \mathrm{~s}\left(1 \mathrm{H}\right.$, endo- $\left.\mathrm{CH}-\mathrm{C}_{2}-\mathrm{CH}-\mathrm{NH}\right)$, 
$2.80 \mathrm{~s}\left(1 \mathrm{H}\right.$, exo- $\left.\mathrm{CH}-\mathrm{CH}_{2}-\mathrm{CH}-\mathrm{NH}\right), 2.84 \mathrm{~s}(1 \mathrm{H}$, endo$\left.\mathrm{CH}-\mathrm{C}_{2}-\mathrm{CH}-\mathrm{NH}\right), 2.93 \mathrm{~s}\left(1 \mathrm{H}\right.$, exo-CH- $\left.\underline{\mathrm{H}}_{2}-\mathrm{CH}-\mathrm{NH}\right)$, $3.46-3.51 \mathrm{~m}(1 \mathrm{H}$, endo- $\mathrm{C} \underline{\mathrm{H}}-\mathrm{NH}), 4.24$ heptet $(1 \mathrm{H}$, exo-Cㅍ-NH, J $3.9 \mathrm{~Hz}), 5.74 \mathrm{~d}(1 \mathrm{H}$, exo-NH, $J 8.2 \mathrm{~Hz})$, $6.03 \mathrm{q}(1 \mathrm{H}$, endo- $\mathrm{C} \underline{\mathrm{H}}=\mathrm{CH}-\mathrm{CH}-\mathrm{CH}-\mathrm{NH}, J 3.3 \mathrm{~Hz})$, $6.08 \mathrm{q}(1 \mathrm{H}$, exo- $\mathrm{CH}=\mathrm{CH}-\mathrm{CH}-\mathrm{CH}-\mathrm{NH}, J 3.1 \mathrm{~Hz}), 6.17 \mathrm{q}$ $(1 \mathrm{H}$, endo- $\mathrm{CH}=\mathrm{C} \underline{\mathrm{H}}-\mathrm{CH}-\mathrm{CH}-\mathrm{NH}, J 2.8 \mathrm{~Hz}), 6.38 \mathrm{q}$ $(1 \mathrm{H}$, exo-CH$=\mathrm{CH}-\mathrm{CH}-\mathrm{CH}-\mathrm{NH}, J 3.0 \mathrm{~Hz}), 6.42 \mathrm{~d}(1 \mathrm{H}$, endo-NH, J 7.2 Hz), 6.65-6.70 m (1H, 6-H Ph), $6.94 \mathrm{~d}$ $(1 \mathrm{H}, 4-\mathrm{H} \mathrm{Ph}, J 8.1 \mathrm{~Hz}), 7.22 \mathrm{q}(1 \mathrm{H}, 5-\mathrm{H} \mathrm{Ph}, J 7.1 \mathrm{~Hz})$, 7.41-7.46 m (1H, 2-H Ph), $8.44 \mathrm{~s}(1 \mathrm{H}$, endo- $\mathrm{NH}-\mathrm{Ph})$, $8.52 \mathrm{~s}\left(1 \mathrm{H}\right.$, exo-NH-Ph). ${ }^{19} \mathrm{~F}$ NMR spectrum (DMSO- $\left.d_{6}\right)$, $\delta$, ppm: -112.41 . Mass spectrum, $m / z\left(I_{\text {rel }}, \%\right): 246(6.5)$ $[M]^{+}, 180$ (66.7) [F-Ph-NH-C(O)-NH-CH= $\left.\mathrm{CH}_{2}\right]^{+}, 137$ (34.0) $[\mathrm{F}-\mathrm{Ph}-\mathrm{NCO}]^{+}, 111(100),\left[\mathrm{F}-\mathrm{Ph}-\mathrm{NH}_{2}\right]^{+}$. Found, \%: C 68.30; $\mathrm{H} 6.15$; N 11.34; F 7.68. $\mathrm{C}_{14} \mathrm{H}_{15} \mathrm{FN}_{2} \mathrm{O}$. Calculated, \%: C 68.28; H 6.14; N 11.37; F 7.71. M 246.29.

1-(Bicyclo[2.2.1]hept-5-en-2-yl)-3-(4-fluorophenyl)urea (4b) was prepared similarly to compound $\mathbf{4 a}$ from $0.2 \mathrm{~g}(1.48 \mathrm{mmol})$ of bicyclo[2.2.1]hept-5-en-2-yl isocyanate $(2), 0.164 \mathrm{~g}(1.48 \mathrm{mmol})$ of 4-fluoroaniline (3b), and $0.2 \mathrm{~mL}(1.48 \mathrm{mmol})$ of triethylamine. Yield $0.221 \mathrm{~g}(60 \%), \mathrm{mp} 167-168^{\circ} \mathrm{C} .{ }^{1} \mathrm{H}$ NMR spectrum (DMSO- $\left.d_{6}\right), \delta$, ppm: 0.61 d.t $\left(1 \mathrm{H}\right.$, endo- $\mathrm{C}-\underline{\mathrm{H}}-\mathrm{CH}_{2}-\mathrm{CH}-$ $\left.\mathrm{NH}, J_{1} 11.8, J_{2} 3.2 \mathrm{~Hz}\right), 1.19$ d.t $\left(1 \mathrm{H}\right.$, exo-C $\underline{\mathrm{H}}-\mathrm{CH}_{2}-$ $\left.\mathrm{CH}-\mathrm{NH}, J_{1} 12.0, J_{2} 3.4 \mathrm{~Hz}\right), 1.33 \mathrm{q}\left(2 \mathrm{H}\right.$, endo- $\mathrm{CH}_{2}, J$ $8.4 \mathrm{~Hz}), 1.57 \mathrm{q}\left(2 \mathrm{H}\right.$, exo- $\left.\mathrm{CH}_{2}, J 8.4 \mathrm{~Hz}\right), 2.12$ d.d.d $(1 \mathrm{H}$, $\left.\mathrm{CH}-\mathrm{CH}-\mathrm{NH}, J_{1} 12.2, J_{2} 8.8, J_{3} 3.8 \mathrm{~Hz}\right), 2.68 \mathrm{~s}(1 \mathrm{H}$, endo$\left.\mathrm{CH}-\mathrm{CH}_{2}-\mathrm{CH}-\mathrm{NH}\right), 2.80 \mathrm{~s}\left(1 \mathrm{H}\right.$, exo-CH- $\left.\underline{\mathrm{C}}_{2}-\mathrm{CH}-\mathrm{NH}\right)$, $2.83 \mathrm{~s}\left(1 \mathrm{H}\right.$, endo- $\left.\mathrm{CH}-\mathrm{CH}_{2}-\mathrm{CH}-\mathrm{NH}\right), 2.92 \mathrm{~s}(1 \mathrm{H}$, exo$\left.\mathrm{CH}-\mathrm{CH}_{2}-\mathrm{CH}-\mathrm{NH}\right), 3.47-3.52 \mathrm{~m}(1 \mathrm{H}$, endo- $\mathrm{C}-\mathrm{H}-\mathrm{NH})$, 4.24 heptet $(1 \mathrm{H}$, exo-C$-\mathrm{HH}, J 3.9 \mathrm{~Hz}), 5.67 \mathrm{~d}(1 \mathrm{H}$, exo-NH, $J 8.3 \mathrm{~Hz}), 6.04 \mathrm{q}(1 \mathrm{H}$, endo- $\mathrm{C} \underline{\mathrm{H}}=\mathrm{CH}-\mathrm{CH}-\mathrm{CH}-$ $\mathrm{NH}, J 3.3 \mathrm{~Hz}), 6.08 \mathrm{q}(1 \mathrm{H}$, exo- $\underline{\mathrm{H}}=\mathrm{CH}-\mathrm{CH}-\mathrm{CH}-\mathrm{NH}$, $J 3.1 \mathrm{~Hz}), 6.16 \mathrm{q}(1 \mathrm{H}$, endo- $\mathrm{CH}=\mathrm{C} \underline{\mathrm{H}}-\mathrm{CH}-\mathrm{CH}-\mathrm{NH}, J$ $2.8 \mathrm{~Hz}), 6.35 \mathrm{~d}(1 \mathrm{H}$, endo- $\mathrm{NH}, J 7.2 \mathrm{~Hz}), 6.38 \mathrm{q}(1 \mathrm{H}$, exo- $\mathrm{CH}=\mathrm{CH}-\mathrm{CH}-\mathrm{CH}-\mathrm{NH}, J 3.0 \mathrm{~Hz}), 7.02-7.07 \mathrm{~m}(2 \mathrm{H}$, 3,5-H Ph), 7.34-7.41 m(2H, 2,6-H Ph), $8.25 \mathrm{~s}(1 \mathrm{H}$, endo$\mathrm{NH}-\mathrm{Ph}), 8.33 \mathrm{~s}\left(1 \mathrm{H}\right.$, exo-NH-Ph). ${ }^{13} \mathrm{C}$ NMR spectrum (DMSO- $d_{6}$ ), $\delta$, ppm: 34.30 (endo- $\mathrm{CH}_{2}-\mathrm{CH}-\mathrm{NH}$ ), 35.15 (exo- $\mathrm{CH}_{2}-\mathrm{CH}-\mathrm{NH}$ ), 40.41 (endo- $\mathrm{CH}-\mathrm{CH}_{2}-\mathrm{CH}-\mathrm{NH}$ ), 42.09 (exo- $\left.\mathrm{CH}-\mathrm{CH}_{2}-\mathrm{CH}-\mathrm{NH}\right), 45.62$ (endo- $\mathrm{CH}-\mathrm{NH}$ ), 45.98 (exo-CH-NH), 47.89 (2C, endo- + exo- $\mathrm{CH}_{2}$ bridge), 48.95 (endo- $\underline{\mathrm{C}} \mathrm{H}-\mathrm{CH}-\mathrm{NH}$ ), 49.64 (exo- $\mathrm{CH}-\mathrm{CH}-\mathrm{NH}$ ), $115.13 \mathrm{~d}(2 \mathrm{C}, 3,5-\mathrm{C} \mathrm{Ph}, J 22.5 \mathrm{~Hz}), 118.91 \mathrm{~d}(2 \mathrm{C}, 2,6-\mathrm{C}$ $\mathrm{Ph}, J 7.5 \mathrm{~Hz}$ ), 132.03 (endo- $\underline{\mathrm{CH}}=\mathrm{CH}), 134.72$ (endo$\mathrm{CH}=\underline{\mathrm{CH}}), 136.88(1-\mathrm{C} \mathrm{Ph}), 138.57$ (exo- $\underline{\mathrm{CH}}=\mathrm{CH}), 139.10$ $($ exo- $\mathrm{CH}=\underline{\mathrm{CH}}), 154.88[\mathrm{NH}-\mathrm{C}(\mathrm{O})-\mathrm{NH}], 157.83(\mathrm{C}-\mathrm{F})$.
${ }^{19} \mathrm{~F}$ NMR spectrum (DMSO- $\left.d_{6}\right), \delta$, ppm: -122.73 . Mass spectrum, $m / z\left(I_{\text {rel }}, \%\right): 246(4.5)[M]^{+}, 180(92.7)[\mathrm{F}-\mathrm{Ph}-$ $\left.\mathrm{NH}-\mathrm{C}(\mathrm{O})-\mathrm{NH}-\mathrm{CH}=\mathrm{CH}_{2}\right]^{+}, 137$ (50.0) $[\mathrm{F}-\mathrm{Ph}-\mathrm{NCO}]^{+}$, 111 (100) [F-Ph- $\left.\mathrm{NH}_{2}\right]^{+}$. Found, \%: C 68.31; H 6.16; $\mathrm{N}$ 11.35; F 7.70. $\mathrm{C}_{14} \mathrm{H}_{15} \mathrm{FN}_{2} \mathrm{O}$. Calculated, \%: C 68.28; H 6.14; N 11.37; F 7.71. M 246.29.

1-(Bicyclo[2.2.1]hept-5-en-2-yl)-3-(3-chlorophenyl)urea (4c) was prepared similarly to compound $4 \mathbf{a}$ from $0.2 \mathrm{~g}$ (1.48 mmol) of bicyclo[2.2.1] hept-5-en-2-yl isocyanate $(2), 0.188 \mathrm{~g}(1.48 \mathrm{mmol})$ of 3 -chloroaniline (3c), and $0.2 \mathrm{~mL}(1.48 \mathrm{mmol})$ of triethylamine. Yield $0.185 \mathrm{~g}(47 \%), \mathrm{mp} 133-134^{\circ} \mathrm{C} .{ }^{1} \mathrm{H}$ NMR spectrum (DMSO- $\left.d_{6}\right), \delta$, ppm: 0.62 d.t $\left(1 \mathrm{H}\right.$, endo- $\mathrm{C} \underline{\mathrm{H}}-\mathrm{CH}_{2}-\mathrm{CH}-$ $\left.\mathrm{NH}, J_{1} 11.8, J_{2} 3.2 \mathrm{~Hz}\right), 1.18$ d.t $\left(1 \mathrm{H}\right.$, exo-C $\underline{\mathrm{H}}-\mathrm{CH}_{2}-$ $\left.\mathrm{CH}-\mathrm{NH}, J_{1} 12.0, J_{2} 3.4 \mathrm{~Hz}\right), 1.34 \mathrm{q}\left(2 \mathrm{H}\right.$, endo- $\mathrm{CH}_{2}, J$ $8.4 \mathrm{~Hz}), 1.57 \mathrm{q}\left(2 \mathrm{H}\right.$, exo- $\left.\mathrm{CH}_{2}, J 8.4 \mathrm{~Hz}\right), 2.12$ d.d.d $(1 \mathrm{H}$, $\left.\mathrm{CH}-\mathrm{CH}-\mathrm{NH}, J_{1} 12.2, J_{2} 8.8, J_{3} 3.8 \mathrm{~Hz}\right), 2.68 \mathrm{~s}(1 \mathrm{H}$, endo$\left.\mathrm{CH}-\underline{\mathrm{C}}_{2}-\mathrm{CH}-\mathrm{NH}\right), 2.80 \mathrm{~s}\left(1 \mathrm{H}\right.$, exo-CH- $\left.\underline{\mathrm{H}}_{2}-\mathrm{CH}-\mathrm{NH}\right)$, $2.83 \mathrm{~s}\left(1 \mathrm{H}\right.$, endo- $\left.\mathrm{CH}-\mathrm{CH}_{2}-\mathrm{CH}-\mathrm{NH}\right), 2.92 \mathrm{~s}(1 \mathrm{H}$, exo$\left.\mathrm{CH}-\mathrm{CH}_{2}-\mathrm{CH}-\mathrm{H}\right), 3.47-3.52 \mathrm{~m}(1 \mathrm{H}$, endo-C $\underline{\mathrm{H}}-\mathrm{NH}), 4.24$ heptet $(1 \mathrm{H}$, exo-C $\underline{\mathrm{H}}-\mathrm{NH}, J 3.9 \mathrm{~Hz}), 5.77 \mathrm{~d}(1 \mathrm{H}$, exo-NH, $J 8.3 \mathrm{~Hz}), 6.03 \mathrm{q}(1 \mathrm{H}$, endo- $\underline{\mathrm{H}}=\mathrm{CH}-\mathrm{CH}-\mathrm{CH}-\mathrm{NH}, J$ $3.3 \mathrm{~Hz}), 6.08$ q $(1 \mathrm{H}$, exo $-\mathrm{CH}=\mathrm{CH}-\mathrm{CH}-\mathrm{CH}-\mathrm{NH}, J$ $3.1 \mathrm{~Hz}), 6.17 \mathrm{q}(1 \mathrm{H}$, endo- $\mathrm{CH}=\mathrm{C} \underline{H}-\mathrm{CH}-\mathrm{CH}-\mathrm{NH}, J$ $2.8 \mathrm{~Hz}), 6.38$ q $(1 \mathrm{H}$, exo- $\mathrm{CH}=\mathrm{CH}-\mathrm{CH}-\mathrm{CH}-\mathrm{NH}, J$ $3.0 \mathrm{~Hz}), 6.49 \mathrm{~d}(1 \mathrm{H}$, endo-NH, $J 7.2 \mathrm{~Hz}), 6.90-6.94 \mathrm{~m}$ (1H, 4-H Ph), 7.20-7.24 m (2H, 5,6-H Ph), 7.66 t $(1 \mathrm{H}$, $2-\mathrm{H} \mathrm{Ph}, J 2.0 \mathrm{~Hz}), 8.48 \mathrm{~s}(1 \mathrm{H}$, endo-NH-Ph), $8.53 \mathrm{~s}$ (1H, exo-NH-Ph). Found, \%: C 64.03; H 5.73; N 10.70. $\mathrm{C}_{14} \mathrm{H}_{15} \mathrm{ClN}_{2} \mathrm{O}$. Calculated, \%: C 64.00; H 5.75; N 10.66. M 62.74.

1-(Bicyclo[2.2.1] hept-5-en-2-yl)-3-(2,4-difluorophenyl)urea (4d) was prepared similarly to compound $\mathbf{4 a}$ from $0.2 \mathrm{~g}$ (1.48 mmol) of bicyclo [2.2.1]hept-5-en-2-yl isocyanate $(2), 0.191 \mathrm{~g}(1.48 \mathrm{mmol})$ of 2,4-difluoroaniline $(\mathbf{3 d})$, and $0.2 \mathrm{~mL}(1.48 \mathrm{mmol})$ of triethylamine. Yield $0.154 \mathrm{~g}(39 \%), \mathrm{mp} 121-122^{\circ} \mathrm{C} .{ }^{1} \mathrm{H}$ NMR spectrum (DMSO- $\left.d_{6}\right), \delta$, ppm: 0.61 d.t $\left(1 \mathrm{H}\right.$, endo- $\mathrm{C}-\underline{\mathrm{H}}-\mathrm{CH}_{2}-\mathrm{CH}-$ $\left.\mathrm{NH}, J_{1} 11.8, J_{2} 3.2 \mathrm{~Hz}\right), 1.18$ d.t $\left(1 \mathrm{H}\right.$, exo- $\mathrm{C} \underline{\mathrm{H}}-\mathrm{CH}_{2}-$ CH-NH, $\left.J_{1} 12.0, J_{2} 3.4 \mathrm{~Hz}\right), 1.34 \mathrm{q}\left(2 \mathrm{H}\right.$, endo- $\mathrm{CH}_{2}, J$ $8.4 \mathrm{~Hz}), 1.45 \mathrm{q}\left(2 \mathrm{H}\right.$, exo $\left.-\mathrm{CH}_{2}, J 8.4 \mathrm{~Hz}\right), 2.12$ d.d.d $(1 \mathrm{H}$, C $\left.\underline{H}-\mathrm{CH}-\mathrm{NH}, J_{1} 12.2, J_{2} 8.8, J_{3} 3.8 \mathrm{~Hz}\right), 2.68 \mathrm{~s}(1 \mathrm{H}$, endo$\left.\mathrm{CH}-\mathrm{CH}_{2}-\mathrm{CH}-\mathrm{NH}\right), 2.80 \mathrm{~s}\left(1 \mathrm{H}\right.$, exo-CH- $\left.\mathrm{CH}_{2}-\mathrm{CH}-\mathrm{NH}\right)$, $2.83 \mathrm{~s}\left(1 \mathrm{H}\right.$, endo- $\left.\mathrm{CH}-\mathrm{CH}_{2}-\mathrm{CH}-\mathrm{NH}\right), 2.92 \mathrm{~s}(1 \mathrm{H}$, exo$\left.\mathrm{CH}-\mathrm{CH}_{2}-\mathrm{CH}-\mathrm{NH}\right), 3.47-3.52 \mathrm{~m}(1 \mathrm{H}$, endo- $\underline{\mathrm{H}}-\mathrm{NH})$, 4.24 heptet $(1 \mathrm{H}$, exo-C$-\mathrm{H}-\mathrm{NH}, J 3.9 \mathrm{~Hz}), 6.03 \mathrm{q}(1 \mathrm{H}$, endo- $\mathrm{C} \underline{\mathrm{H}}=\mathrm{CH}-\mathrm{CH}-\mathrm{CH}-\mathrm{NH}, J 3.3 \mathrm{~Hz}), 6.08 \mathrm{q}(1 \mathrm{H}$, exo- $\mathrm{C} \underline{\mathrm{H}}=\mathrm{CH}-\mathrm{CH}-\mathrm{CH}-\mathrm{NH}, J 3.1 \mathrm{~Hz}), 6.17 \mathrm{q}(1 \mathrm{H}$, endo- 
$\mathrm{CH}=\mathrm{C} \underline{\mathrm{H}}-\mathrm{CH}-\mathrm{CH}-\mathrm{NH}, J 2.8 \mathrm{~Hz}), 6.20 \mathrm{~d}(1 \mathrm{H}$, exo-NH, $J 8.2 \mathrm{~Hz}), 6.38$ q $(1 \mathrm{H}$, exo-CH$=\mathrm{C} \underline{\mathrm{H}}-\mathrm{CH}-\mathrm{CH}-\mathrm{NH}, J$ $3.0 \mathrm{~Hz}), 6.80 \mathrm{~d}(1 \mathrm{H}$, endo-NH, J 7.2 Hz), 6.93-7.00 m (1H, 3-H Ph), 7.17-7.25 m (2H, 5-H Ph), 8.07-8.14 m $(1 \mathrm{H}, 6-\mathrm{H} \mathrm{Ph}), 8.17 \mathrm{~s}(1 \mathrm{H}$, exo-NH-Ph $), 8.91 \mathrm{~s}(1 \mathrm{H}$, endo-NH-Ph). ${ }^{19} \mathrm{~F}$ NMR spectrum (DMSO- $\left.d_{6}\right), \delta$, ppm: -126.40 (exo-4-F), -124.77 (endo-4-F), -120.00 (exo$2-\mathrm{F}),-117.99$ (endo-2-F). Mass spectrum, $m / z\left(I_{\text {rel }}, \%\right)$ : $264(5.0)[M]^{+}, 198(40.6)\left[\mathrm{F}_{2}-\mathrm{Ph}-\mathrm{NH}-\mathrm{C}(\mathrm{O})-\mathrm{NH}-\right.$ $\left.\mathrm{CH}=\mathrm{CH}_{2}\right]^{+}, 155(10.0)\left[\mathrm{F}_{2}-\mathrm{Ph}-\mathrm{NCO}\right]^{+}, 129(100)\left[\mathrm{F}_{2}-\right.$ $\left.\mathrm{Ph}-\mathrm{NH}_{2}\right]^{+}$. Found, \%: C 63.60; H 5.36; N 10.63; F 14.34. $\mathrm{C}_{14} \mathrm{H}_{14} \mathrm{~F}_{2} \mathrm{~N}_{2} \mathrm{O}$. Calculated, \%: C 63.63; H 5.34; N 10.60; F 14.38. $M$ 264.28.

1-(Bicyclo[2.2.1]hept-5-en-2-yl)-3-(2,6-difluorophenyl)urea (4e) was prepared similarly to compound $\mathbf{4 a}$ from $0.2 \mathrm{~g}(1.48 \mathrm{mmol})$ of bicyclo[2.2.1]hept-5-en-2-yl isocyanate (2), $0.191 \mathrm{~g}$ (1.48 mmol) of 2,6-difluoroaniline (3e), and $0.2 \mathrm{~mL}(1.48 \mathrm{mmol})$ of triethylamine. Yield $0.1 \mathrm{~g}(26 \%), \mathrm{mp} 170-171^{\circ} \mathrm{C} .{ }^{1} \mathrm{H}$ NMR spectrum (DMSO$\left.d_{6}\right), \delta$, ppm: 0.64 d.t $\left(1 \mathrm{H}\right.$, endo- $\mathrm{C} \underline{\mathrm{H}}-\mathrm{CH}_{2}-\mathrm{CH}-\mathrm{NH}, J_{1}$ $\left.11.8, J_{2} 3.2 \mathrm{~Hz}\right), 1.10$ d.t $\left(1 \mathrm{H}\right.$, exo- $\underline{\mathrm{H}}-\mathrm{CH}_{2}-\mathrm{CH}-\mathrm{NH}, J_{1}$ $\left.12.0, J_{2} 3.4 \mathrm{~Hz}\right), 1.30 \mathrm{q}\left(2 \mathrm{H}\right.$, endo- $\left.\mathrm{CH}_{2}, J 8.4 \mathrm{~Hz}\right), 1.46 \mathrm{q}$ $\left(2 \mathrm{H}\right.$, exo- $\left.\mathrm{CH}_{2}, J 8.4 \mathrm{~Hz}\right), 2.12$ d.d.d $\left(1 \mathrm{H}, \mathrm{CH}-\mathrm{CH}-\mathrm{NH}, J_{1}\right.$ $\left.12.2, J_{2} 8.8, J_{3} 3.8 \mathrm{~Hz}\right), 2.67 \mathrm{~s}\left(1 \mathrm{H}\right.$, endo- $\mathrm{CH}-\mathrm{CH}_{2}-\mathrm{CH}-$ $\mathrm{NH}), 2.74 \mathrm{~s}\left(1 \mathrm{H}\right.$, exo-CH- $\left.\underline{\mathrm{H}}_{2}-\mathrm{CH}-\mathrm{NH}\right), 2.80 \mathrm{~s}(1 \mathrm{H}$, endo- $\left.\mathrm{CH}-\mathrm{CH}_{2}-\mathrm{CH}-\mathrm{NH}\right), 2.93 \mathrm{~s}\left(1 \mathrm{H}\right.$, exo- $\mathrm{CH}-\mathrm{CH}_{2}-$ $\mathrm{CH}-\mathrm{NH}), 3.47-3.52 \mathrm{~m}(1 \mathrm{H}$, endo-CH-NH), 4.20 heptet $(1 \mathrm{H}$, exo-C $\underline{\mathrm{H}}-\mathrm{NH}, J 3.9 \mathrm{~Hz}), 5.85 \mathrm{~d}(1 \mathrm{H}$, exo-NH, $J$ $8.2 \mathrm{~Hz}), 6.03$ q $(1 \mathrm{H}$, endo- $\underline{\mathrm{H}}=\mathrm{CH}-\mathrm{CH}-\mathrm{CH}-\mathrm{NH}, J$ $3.3 \mathrm{~Hz}), 6.08 \mathrm{q}(1 \mathrm{H}$, exo $-\mathrm{C} \underline{\mathrm{H}}=\mathrm{CH}-\mathrm{CH}-\mathrm{CH}-\mathrm{NH}, J$ $3.1 \mathrm{~Hz}), 6.17 \mathrm{q}(1 \mathrm{H}$, endo- $\mathrm{CH}=\mathrm{CH}-\mathrm{CH}-\mathrm{CH}-\mathrm{NH}, J$ $2.8 \mathrm{~Hz}), 6.38$ q $(1 \mathrm{H}$, exo- $\mathrm{CH}=\mathrm{C} \underline{\mathrm{H}}-\mathrm{CH}-\mathrm{CH}-\mathrm{NH}, J$ $3.0 \mathrm{~Hz}), 6.56 \mathrm{~d}(1 \mathrm{H}$, endo-NH, J 7.2 Hz), 7.05-7.10 m $(2 \mathrm{H}, 3,5-\mathrm{H} \mathrm{Ph}), 7.18-7.24 \mathrm{~m}(1 \mathrm{H}, 4-\mathrm{H} \mathrm{Ph}), 7.62 \mathrm{~s}(1 \mathrm{H}$, endo-NH-Ph), $7.69 \mathrm{~s}\left(1 \mathrm{H}\right.$, exo-NH-Ph). ${ }^{19} \mathrm{~F}$ NMR spectrum (DMSO- $\left.d_{6}\right), \delta$, ppm: $-119.01(2 \mathrm{~F})$. Mass spectrum, $m / z\left(I_{\text {rel }}, \%\right): 264(0.5)[M]^{+}, 198(46.8)\left[\mathrm{F}_{2}-\mathrm{Ph}-\right.$ $\left.\mathrm{NH}-\mathrm{C}(\mathrm{O})-\mathrm{NH}-\mathrm{CH}=\mathrm{CH}_{2}\right]^{+}, 155(6.5)\left[\mathrm{F}_{2}-\mathrm{Ph}-\mathrm{NCO}\right]^{+}$, 129 (100) $\left[\mathrm{F}_{2}-\mathrm{Ph}-\mathrm{NH}_{2}\right]^{+}$. Found, \%: C 63.61; H 5.35; $\mathrm{N}$ 10.63; F 14.35. $\mathrm{C}_{14} \mathrm{H}_{14} \mathrm{~F}_{2} \mathrm{~N}_{2} \mathrm{O}$. Calculated, \%: C 63.63; H 5.34; N 10.60; F 14.38. M 264.28.

1-(Bicyclo[2.2.1]hept-5-en-2-yl)-3-(3,4-difluorophenyl)urea (4f) was prepared similarly to compound $\mathbf{4 a}$ from $0.2 \mathrm{~g}$ (1.48 mmol) of bicyclo[2.2.1] hept-5-en-2-yl isocyanate $(2), 0.191 \mathrm{~g}(1.48 \mathrm{mmol})$ of 3,4-difluoroaniline $(\mathbf{3 f})$, and $0.2 \mathrm{~mL}(1.48 \mathrm{mmol})$ of triethylamine. Yield $0.241 \mathrm{~g}(61 \%), \mathrm{mp} 143-144^{\circ} \mathrm{C} .{ }^{1} \mathrm{H}$ NMR spectrum $\left(\mathrm{DMSO}-d_{6}\right), \delta$, ppm: 0.62 d.t $\left(1 \mathrm{H}\right.$, endo- $\mathrm{C}-\mathrm{H}-\mathrm{CH}_{2}-\mathrm{CH}-$
$\left.\mathrm{NH}, J_{1} 11.8, J_{2} 3.2 \mathrm{~Hz}\right), 1.18$ d.t $\left(1 \mathrm{H}\right.$, exo-C $\underline{\mathrm{H}}-\mathrm{CH}_{2}-$ $\left.\mathrm{CH}-\mathrm{NH}, J_{1} 12.0, J_{2} 3.4 \mathrm{~Hz}\right), 1.33 \mathrm{q}\left(2 \mathrm{H}\right.$, endo- $\mathrm{CH}_{2}, J$ $8.4 \mathrm{~Hz}), 1.56 \mathrm{q}\left(2 \mathrm{H}\right.$, exo- $\left.\mathrm{CH}_{2}, J 8.4 \mathrm{~Hz}\right), 2.12$ d.d.d $(1 \mathrm{H}$, $\left.\mathrm{C} \underline{\mathrm{H}}-\mathrm{CH}-\mathrm{NH}, J_{1} 12.2, J_{2} 8.8, J_{3} 3.8 \mathrm{~Hz}\right), 2.67 \mathrm{~s}(1 \mathrm{H}$, endo$\left.\mathrm{CH}-\mathrm{CH}_{2}-\mathrm{CH}-\mathrm{NH}\right), 2.80 \mathrm{~s}\left(1 \mathrm{H}\right.$, exo-CH- $\left.\underline{\mathrm{H}}_{2}-\mathrm{CH}-\mathrm{NH}\right)$, $2.82 \mathrm{~s}\left(1 \mathrm{H}\right.$, endo- $\left.\mathrm{CH}-\mathrm{CH}_{2}-\mathrm{CH}-\mathrm{NH}\right), 2.93 \mathrm{~s}(1 \mathrm{H}$, exo$\left.\mathrm{CH}-\mathrm{C}_{2}-\mathrm{CH}-\mathrm{NH}\right), 3.46-3.50 \mathrm{~m}(1 \mathrm{H}$, endo- $\mathrm{C} \underline{\mathrm{H}}-\mathrm{NH})$, 4.23 heptet $(1 \mathrm{H}$, exo-C$-\mathrm{HH}, J 3.9 \mathrm{~Hz}), 5.75 \mathrm{~d}(1 \mathrm{H}$, exo- $\mathrm{NH}, J 8.2 \mathrm{~Hz}), 6.03 \mathrm{q}(1 \mathrm{H}$, endo- $\mathrm{CH}=\mathrm{CH}-\mathrm{CH}-\mathrm{CH}-$ $\mathrm{NH}, J 3.3 \mathrm{~Hz}), 6.08 \mathrm{q}(1 \mathrm{H}$, exo- $\mathrm{C} \underline{\mathrm{H}}=\mathrm{CH}-\mathrm{CH}-\mathrm{CH}-\mathrm{NH}, J$ $3.1 \mathrm{~Hz}), 6.17$ q $(1 \mathrm{H}$, endo- $\mathrm{CH}=\mathrm{CH}-\mathrm{CH}-\mathrm{CH}-\mathrm{NH}, J$ $2.8 \mathrm{~Hz}), 6.38 \mathrm{q}(1 \mathrm{H}$, exo- $\mathrm{CH}=\mathrm{C} \underline{\mathrm{H}}-\mathrm{CH}-\mathrm{CH}-\mathrm{NH}, J$ $3.0 \mathrm{~Hz}), 6.48 \mathrm{~d}(1 \mathrm{H}$, endo-NH, J 7.2 Hz), 6.92-6.96 m (1H, 6-H Ph), 7.22-7.30 m (1H, 5-H Ph), 7.59-7.66 m $(1 \mathrm{H}, 5-\mathrm{H} \mathrm{Ph}), 8.50 \mathrm{~s}(1 \mathrm{H}$, endo-NH-Ph $), 8.55 \mathrm{~s}(1 \mathrm{H}$, exo-NH-Ph). ${ }^{19} \mathrm{~F}$ NMR spectrum (DMSO- $\left.d_{6}\right), \delta$, ppm: $-148.9,-137.70$. Mass spectrum, $m / z\left(I_{\text {rel }}, \%\right): 264$ (2.7) $[M]^{+}, 198$ (56.7) $\left[\mathrm{F}_{2}-\mathrm{Ph}-\mathrm{NH}-\mathrm{C}(\mathrm{O})-\mathrm{NH}-\mathrm{CH}=\mathrm{CH}_{2}\right]^{+}$, 155 (36.6) $\left[\mathrm{F}_{2}-\mathrm{Ph}-\mathrm{NCO}\right]^{+}, 129$ (100) $\left[\mathrm{F}_{2}-\mathrm{Ph}-\mathrm{NH}_{2}\right]^{+}$. Found, \%: C 63.64; H 5.37; N 10.62; F 14.39. $\mathrm{C}_{14} \mathrm{H}_{14} \mathrm{~F}_{2} \mathrm{~N}_{2} \mathrm{O}$. Calculated, \%: C 63.63; H 5.34; N 10.60; F 14.38. M 264.28.

1-(Bicyclo[2.2.1]hept-5-en-2-yl)-3-(2,5-difluorophenyl)urea (4g) was prepared similarly to compound 4a from $0.2 \mathrm{~g}(1.48 \mathrm{mmol})$ bicyclo[2.2.1]hept-5-en-2-yl isocyanate $(2), 0.191 \mathrm{~g}(1.48 \mathrm{mmol})$ of 2,5-difluoroaniline $(\mathbf{3 g})$, and $0.2 \mathrm{~mL}(1.48 \mathrm{mmol})$ of triethylamine. Yield $0.183 \mathrm{~g}(47 \%), \mathrm{mp} 146-147^{\circ} \mathrm{C} .{ }^{1} \mathrm{H}$ NMR spectrum DMSO- $\left.d_{6}\right), \delta$, ppm: 0.61 d.t $\left(1 \mathrm{H}\right.$, endo- $\underline{\mathrm{H}}-\mathrm{CH}_{2}-\mathrm{CH}-$ $\left.\mathrm{NH}, J_{1} 11.8, J_{2} 3.2 \mathrm{~Hz}\right), 1.26$ d.t $\left(1 \mathrm{H}\right.$, exo-C $\underline{\mathrm{H}}-\mathrm{CH}_{2}-$ $\left.\mathrm{CH}-\mathrm{NH}, J_{1} 12.0, J_{2} 3.4 \mathrm{~Hz}\right), 1.34 \mathrm{q}\left(2 \mathrm{H}\right.$, endo- $\mathrm{CH}_{2}, J$ $8.4 \mathrm{~Hz}), 1.59 \mathrm{q}\left(2 \mathrm{H}\right.$, exo- $\left.\mathrm{CH}_{2}, J 8.4 \mathrm{~Hz}\right), 2.12$ d.d.d $(1 \mathrm{H}$, $\left.\mathrm{C} \underline{\mathrm{H}}-\mathrm{CH}-\mathrm{NH}, J_{1} 12.2, J_{2} 8.8, J_{3} 3.8 \mathrm{~Hz}\right), 2.69 \mathrm{~s}(1 \mathrm{H}$, endo$\left.\mathrm{CH}-\mathrm{C}_{2}-\mathrm{CH}-\mathrm{NH}\right), 2.81 \mathrm{~s}\left(1 \mathrm{H}\right.$, exo-CH- $\left.\underline{\mathrm{H}}_{2}-\mathrm{CH}-\mathrm{NH}\right)$, $2.85 \mathrm{~s}\left(1 \mathrm{H}\right.$, endo- $\left.\mathrm{CH}-\mathrm{CH}_{2}-\mathrm{CH}-\mathrm{NH}\right), 2.94 \mathrm{~s}(1 \mathrm{H}$, exo$\left.\mathrm{CH}-\underline{\mathrm{C}}_{2}-\mathrm{CH}-\mathrm{NH}\right), 3.46-3.50 \mathrm{~m}(1 \mathrm{H}$, endo- $\mathrm{C} \underline{\mathrm{H}}-\mathrm{NH})$, 4.24 heptet $(1 \mathrm{H}$, exo-Cㅍ- $-\mathrm{NH}, J 3.9 \mathrm{~Hz}), 5.28 \mathrm{~d}(1 \mathrm{H}$, exo-NH, $J 8.2 \mathrm{~Hz}), 6.03 \mathrm{q}(1 \mathrm{H}$, endo- $\mathrm{CH}=\mathrm{CH}-\mathrm{CH}-\mathrm{CH}-$ $\mathrm{NH}, J 3.3 \mathrm{~Hz}), 6.08 \mathrm{q}(1 \mathrm{H}$, exo- $\mathrm{C} \underline{\mathrm{H}}=\mathrm{CH}-\mathrm{CH}-\mathrm{CH}-\mathrm{NH}$, $J 3.1 \mathrm{~Hz}), 6.17$ q $(1 \mathrm{H}$, endo- $\mathrm{CH}=\mathrm{C} \underline{\mathrm{H}}-\mathrm{CH}-\mathrm{CH}-\mathrm{NH}, J$ $2.8 \mathrm{~Hz}), 6.38 \mathrm{q}(1 \mathrm{H}$, exo- $\mathrm{CH}=\mathrm{C} \underline{\mathrm{H}}-\mathrm{CH}-\mathrm{CH}-\mathrm{NH}, J$ $3.0 \mathrm{~Hz}), 6.95 \mathrm{~d}(1 \mathrm{H}$, endo-NH, J 7.2 Hz), 7.17-7.24 m (1H, 4-H Ph), 7.28-7.34 m (1H, 3-H Ph), 8.02-8.07 m $(1 \mathrm{H}, 6-\mathrm{H} \mathrm{Ph}), 8.34 \mathrm{~s}(1 \mathrm{H}$, endo-NH-Ph $), 8.42 \mathrm{~s}(1 \mathrm{H}$, exo-NH-Ph). ${ }^{19} \mathrm{~F}$ NMR spectrum (DMSO- $\left.d_{6}\right), \delta$, ppm: -136.52 (endo-5-F), -136.07 (exo-4-F), -116.97 (endo$2-\mathrm{F}),-116.66$ (exo-2-F). Mass spectrum, $m / z\left(I_{\text {rel }}, \%\right)$ :

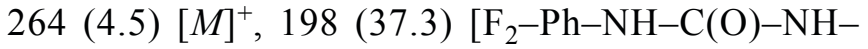


$\left.\mathrm{CH}=\mathrm{CH}_{2}\right]^{+}, 155(8.0)\left[\mathrm{F}_{2}-\mathrm{Ph}-\mathrm{NCO}\right]^{+}, 129(100)\left[\mathrm{F}_{2}-\right.$ $\left.\mathrm{Ph}-\mathrm{NH}_{2}\right]^{+}$. Found, \%: C 63.67; H 5.38; N 10.59; F 14.34. $\mathrm{C}_{14} \mathrm{H}_{14} \mathrm{~F}_{2} \mathrm{~N}_{2} \mathrm{O}$. Calculated, \%: C 63.63; H 5.34; N 10.60; F 14.38. M 264.28.

1-(Bicyclo[2.2.1]hept-5-en-2-yl)-3-(3,5-difluorophenyl)urea (4h) was prepared similarly to compound $\mathbf{4 a}$ from $0.2 \mathrm{~g}(1.48 \mathrm{mmol})$ of bicyclo[2.2.1]hept-5-en-2-yl isocyanate $(2), 0.191 \mathrm{~g}(1.48 \mathrm{mmol})$ of 3,5-difluoroaniline $(\mathbf{3 h})$, and $0.2 \mathrm{~mL}(1.48 \mathrm{mmol})$ of triethylamine. Yield $0.116 \mathrm{~g}(29 \%), \mathrm{mp} 143-144^{\circ} \mathrm{C} .{ }^{1} \mathrm{H}$ NMR spectrum $\left(\mathrm{DMSO}-d_{6}\right), \delta$, ppm: 0.63 d.t $\left(1 \mathrm{H}\right.$, endo- $\mathrm{C} \underline{\mathrm{H}}-\mathrm{CH}_{2}-\mathrm{CH}-$ $\left.\mathrm{NH}, J_{1} 11.8, J_{2} 3.2 \mathrm{~Hz}\right), 1.26$ d.t $\left(1 \mathrm{H}\right.$, exo- $\overline{\mathrm{H}}-\mathrm{CH}_{2}-\mathrm{CH}-$ $\left.\mathrm{NH}, J_{1} 12.0, J_{2} 3.4 \mathrm{~Hz}\right), 1.34 \mathrm{q}\left(2 \mathrm{H}\right.$, endo $\left.-\mathrm{CH}_{2}, J 8.4 \mathrm{~Hz}\right)$, $1.57 \mathrm{q}\left(2 \mathrm{H}\right.$, exo- $\left.\mathrm{CH}_{2}, J 8.4 \mathrm{~Hz}\right), 2.12$ d.d.d $(1 \mathrm{H}, \mathrm{C} \underline{\mathrm{H}}-\mathrm{CH}-$ $\left.\mathrm{NH}, J_{1} 12.2, J_{2} 8.8, J_{3} 3.8 \mathrm{~Hz}\right), 2.69 \mathrm{~s}\left(1 \mathrm{H}\right.$, endo $-\mathrm{CH}-\mathrm{CH}_{2}-$ $\mathrm{CH}-\mathrm{NH}), 2.81 \mathrm{~s}\left(1 \mathrm{H}\right.$, exo-CH- $\left.\underline{\mathrm{H}}_{2}-\mathrm{CH}-\mathrm{NH}\right), 2.85 \mathrm{~s}(1 \mathrm{H}$, endo- $\left.\mathrm{CH}-\mathrm{CH}_{2}-\mathrm{CH}-\mathrm{NH}\right), 2.94 \mathrm{~s}\left(1 \mathrm{H}\right.$, exo- $\mathrm{CH}-\mathrm{CH}_{2}-\mathrm{CH}-$ $\mathrm{NH}), 3.46-3.50 \mathrm{~m}(1 \mathrm{H}$, endo-C $\mathrm{H}-\mathrm{NH}), 4.24$ heptet $(1 \mathrm{H}$, exo-C $\underline{\mathrm{H}}-\mathrm{NH}, J 3.9 \mathrm{~Hz}), 5.85 \mathrm{~d}(1 \mathrm{H}$, exo-NH, $J 8.2 \mathrm{~Hz})$, $6.03 \mathrm{q}(1 \mathrm{H}$, endo- $\mathrm{C}=\mathrm{CH}-\mathrm{CH}-\mathrm{CH}-\mathrm{NH}, J 3.3 \mathrm{~Hz})$, $6.08 \mathrm{q}(1 \mathrm{H}$, exo- $\mathrm{CH}=\mathrm{CH}-\mathrm{CH}-\mathrm{CH}-\mathrm{NH}, J 3.1 \mathrm{~Hz}), 6.17 \mathrm{q}$ $(1 \mathrm{H}$, endo- $\mathrm{CH}=\mathrm{CH}-\mathrm{CH}-\mathrm{CH}-\mathrm{NH}, J 2.8 \mathrm{~Hz}), 6.38 \mathrm{q}(1 \mathrm{H}$, exo- $\mathrm{CH}=\mathrm{CH}-\mathrm{CH}-\mathrm{CH}-\mathrm{NH}, J 3.0 \mathrm{~Hz}), 6.57 \mathrm{~d}(1 \mathrm{H}$, endo$\mathrm{NH}, J 7.2 \mathrm{~Hz}), 6.82$ t.t $\left(1 \mathrm{H}, 4-\mathrm{H} \mathrm{Ph}, J_{1} 11.7, J_{2} 2.3 \mathrm{~Hz}\right)$, 7.20 d.d $\left(2 \mathrm{H}, 2,6-\mathrm{H} \mathrm{Ph}, J_{1} 9.6, J_{2} 2.1 \mathrm{~Hz}\right), 8.65 \mathrm{~s}(1 \mathrm{H}$, endo-NH-Ph), $8.72 \mathrm{~s}\left(1 \mathrm{H}\right.$, exo-NH-Ph). ${ }^{19} \mathrm{~F}$ NMR spectrum (DMSO- $\left.d_{6}\right), \delta$, ppm: $-110.00(2 \mathrm{~F}$, endo-),-109.51 (2F, exo-). Mass spectrum, $m / z\left(I_{\text {rel }}, \%\right): 264$ (6.7) $[M]^{+}, 198(93.3)\left[\mathrm{F}_{2}-\mathrm{Ph}-\mathrm{NH}-\mathrm{C}(\mathrm{O})-\mathrm{NH}-\mathrm{CH}=\mathrm{CH}_{2}\right]^{+}$, 155 (31.1) $\left[\mathrm{F}_{2}-\mathrm{Ph}-\mathrm{NCO}\right]^{+}, 129(100)\left[\mathrm{F}_{2}-\mathrm{Ph}-\mathrm{NH}_{2}\right]^{+}$. Found, \%: C 63.65; H 5.37; N 10.61; F 14.37. $\mathrm{C}_{14} \mathrm{H}_{14} \mathrm{~F}_{2} \mathrm{~N}_{2} \mathrm{O}$. Calculated, \%: C 63.63; H 5.34; N 10.60; F 14.38. $M$ 264.28.

1-(Bicyclo[2.2.1]hept-5-en-2-yl)-3-(2-chloro-4fluorophenyl)urea (4i) was prepared similarly to compound 4a from $0.2 \mathrm{~g}(1.48 \mathrm{mmol})$ of bicyclo[2.2.1]hept-5-en-2-yl isocyanate (2), $0.214 \mathrm{~g}(1.48 \mathrm{mmol})$ of 2-chloro-4-fluoroaniline (3i), and $0.2 \mathrm{~mL}(1.48 \mathrm{mmol})$ of triethylamine. Yield $0.147 \mathrm{~g}(35 \%), \mathrm{mp} 135-136^{\circ} \mathrm{C}$. ${ }^{1} \mathrm{H}$ NMR spectrum (DMSO- $\left.d_{6}\right), \delta$, ppm: 0.64 d.t $(1 \mathrm{H}$, endo-C $\left.\underline{H}-\mathrm{CH} 2-\mathrm{CH}-\mathrm{NH}, J_{1} 11.8, J_{2} 3.2 \mathrm{~Hz}\right), 1.19$ d.t $\left(1 \mathrm{H}\right.$, exo-C $\left.\underline{\mathrm{H}}-\mathrm{CH}_{2}-\mathrm{CH}-\mathrm{NH}, J_{1} 12.0, J_{2} 3.4 \mathrm{~Hz}\right), 1.30 \mathrm{q}$ $\left(2 \mathrm{H}\right.$, endo- $\left.\mathrm{CH}_{2}, J 8.4 \mathrm{~Hz}\right), 1.58 \mathrm{q}\left(2 \mathrm{H}\right.$, exo- $\left.\mathrm{CH}_{2}, J 8.4 \mathrm{~Hz}\right)$, 2.12 d.d.d ( $\left.1 \mathrm{H}, \mathrm{C} \underline{\mathrm{H}}-\mathrm{CH}-\mathrm{NH}, J_{1} 12.2, J_{2} 8.8, J_{3} 3.8 \mathrm{~Hz}\right)$, $2.69 \mathrm{~s}\left(1 \mathrm{H}\right.$, endo- $\left.\mathrm{CH}-\mathrm{CH}_{2}-\mathrm{CH}-\mathrm{NH}\right), 2.81 \mathrm{~s}(1 \mathrm{H}$, exo$\left.\mathrm{CH}-\mathrm{CH}_{2}-\mathrm{CH}-\mathrm{NH}\right), 2.85 \mathrm{~s}\left(1 \mathrm{H}\right.$, endo- $\mathrm{CH}-\mathrm{CH}_{2}-\mathrm{CH}-$ $\mathrm{NH}), 2.94 \mathrm{~s}\left(1 \mathrm{H}\right.$, exo-CH-C $\left.\underline{\mathrm{H}}_{2}-\mathrm{CH}-\mathrm{NH}\right), 3.47-3.52 \mathrm{~m}$ $(1 \mathrm{H}$, endo- $\mathrm{C} \underline{\mathrm{H}}-\mathrm{NH}), 4.24$ heptet $(1 \mathrm{H}$, exo- $\mathrm{C} \underline{\mathrm{H}}-\mathrm{NH}, J$
$3.9 \mathrm{~Hz}), 5.29 \mathrm{~d}(1 \mathrm{H}$, exo-NH, $J 8.2 \mathrm{~Hz}), 6.04 \mathrm{q}(1 \mathrm{H}$, endo- $\mathrm{CH}=\mathrm{CH}-\mathrm{CH}-\mathrm{CH}-\mathrm{NH}, J 3.3 \mathrm{~Hz}), 6.08 \mathrm{q}(1 \mathrm{H}$, exo- $\mathrm{C} \underline{\mathrm{H}}=\mathrm{CH}-\mathrm{CH}-\mathrm{CH}-\mathrm{NH}, J 3.1 \mathrm{~Hz}), 6.17 \mathrm{q}(1 \mathrm{H}$, endo- $\mathrm{CH}=\mathrm{C} \underline{\mathrm{H}}-\mathrm{CH}-\mathrm{CH}-\mathrm{NH}, J 2.8 \mathrm{~Hz}), 6.38 \mathrm{q}(1 \mathrm{H}$, exo-CH=CH-CH-CH-NH, J3.0 Hz), $6.56 \mathrm{~d}(1 \mathrm{H}$, endo$\mathrm{NH}, J 7.2 \mathrm{~Hz}), 7.10-7.17 \mathrm{~m}(1 \mathrm{H}, 5-\mathrm{H} \mathrm{Ph}), 7.35-7.41 \mathrm{~m}$ $(1 \mathrm{H}, 6-\mathrm{H} \mathrm{Ph}), 8.88 \mathrm{~s}(1 \mathrm{H}$, endo-NH-Ph), $8.96 \mathrm{~s}(1 \mathrm{H}$, exo-NH-Ph), 8.12-8.17 m (1H, 3-H Ph). ${ }^{19} \mathrm{~F}$ NMR spectrum (DMSO- $d_{6}$ ), $\delta$, ppm: -120.34 (endo), -118.21 (exo). Mass spectrum, $m / z\left(I_{\text {rel }}, \%\right): 280(5.3)[M]^{+}, 214$ (46.4) $\left[\mathrm{FCl}-\mathrm{Ph}-\mathrm{NH}-\mathrm{C}(\mathrm{O})-\mathrm{NH}-\mathrm{CH}=\mathrm{CH}_{2}\right]^{+}, 171$ (17.8) $[\mathrm{FCl}-\mathrm{Ph}-\mathrm{NCO}]^{+}, 145(100)\left[\mathrm{FCl}-\mathrm{Ph}-\mathrm{NH}_{2}\right]^{+}$. Found, \%: C 59.94; H 5.00; N 10.00; F 6.75. $\mathrm{C}_{14} \mathrm{H}_{14} \mathrm{ClFN}_{2} \mathrm{O}$. Calculated, \%: C 59.90; H 5.03; N 9.98; F 6.77. M 280.73.

1-(Bicyclo[2.2.1]hept-5-en-2-yl)-3-(3-chloro-4-fluorophenyl)urea $(\mathbf{4 j})$ was prepared similarly to compound 4a from $0.2 \mathrm{~g}$ (1.48 mmol) of bicyclo[2.2.1]hept-5-en-2-yl

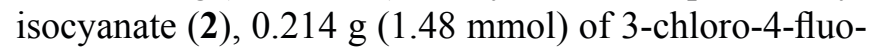
roaniline $(\mathbf{3 j})$, and $0.2 \mathrm{~mL}(1.48 \mathrm{mmol})$ of triethylamine. Yield $0.28 \mathrm{~g}(68 \%), \mathrm{mp} 188-189^{\circ} \mathrm{C} .{ }^{1} \mathrm{H}\left(\mathrm{DMSO}-d_{6}\right)$, $\delta$, ppm: 0.62 d.t $\left(1 \mathrm{H}\right.$, endo- $\underline{\mathrm{H}}-\mathrm{CH}_{2}-\mathrm{CH}-\mathrm{NH}, J_{1} 11.9$, $\left.J_{2} 3.2 \mathrm{~Hz}\right), 1.20$ d.t $\left(1 \mathrm{H}\right.$, exo-C $\underline{\mathrm{H}}-\mathrm{CH}_{2}-\mathrm{CH}-\mathrm{NH}, J_{1} 12.0$, $\left.J_{2} 3.4 \mathrm{~Hz}\right), 1.33 \mathrm{q}\left(2 \mathrm{H}\right.$, endo- $\left.\mathrm{CH}_{2}, J 8.4 \mathrm{~Hz}\right), 1.56 \mathrm{q}(2 \mathrm{H}$, exo- $\left.\mathrm{CH}_{2}, J 8.4 \mathrm{~Hz}\right), 2.12$ d.d.d $\left(1 \mathrm{H}, \mathrm{C} \underline{\mathrm{H}}-\mathrm{CH}-\mathrm{NH}, J_{1} 12.2\right.$, $\left.J_{2} 8.8, J_{3} 3.8 \mathrm{~Hz}\right), 2.69 \mathrm{~s}\left(1 \mathrm{H}\right.$, endo- $\left.\mathrm{CH}-\mathrm{CH}_{2}-\mathrm{CH}-\mathrm{NH}\right)$, $2.80 \mathrm{~s}\left(1 \mathrm{H}\right.$, exo-CH- $\left.\underline{\mathrm{C}}_{2}-\mathrm{CH}-\mathrm{NH}\right), 2.83 \mathrm{~s}(1 \mathrm{H}$, endo$\left.\mathrm{CH}-\mathrm{CH}_{2}-\mathrm{CH}-\mathrm{NH}\right), 2.93 \mathrm{~s}\left(1 \mathrm{H}\right.$, exo- $\left.\mathrm{CH}-\mathrm{CH}_{2}-\mathrm{CH}-\mathrm{NH}\right)$, $3.45-3.50 \mathrm{~m}(1 \mathrm{H}$, endo- $\mathrm{C} \underline{\mathrm{H}}-\mathrm{NH}), 4.23$ heptet $(1 \mathrm{H}$, exoCㅍ-NH, J3.6 Hz), $5.74 \mathrm{~d}(1 \mathrm{H}$, exo-NH, $J 8.2 \mathrm{~Hz}), 6.03 \mathrm{q}$ $(1 \mathrm{H}$, endo- $\mathrm{CH}=\mathrm{CH}-\mathrm{CH}-\mathrm{CH}-\mathrm{NH}, J 3.3 \mathrm{~Hz}), 6.08 \mathrm{q}$ $(1 \mathrm{H}$, exo- $\mathrm{C} \underline{\mathrm{H}}=\mathrm{CH}-\mathrm{CH}-\mathrm{CH}-\mathrm{NH}, J 3.1 \mathrm{~Hz}), 6.17 \mathrm{q}(1 \mathrm{H}$, endo- $\mathrm{CH}=\mathrm{C} \underline{\mathrm{H}}-\mathrm{CH}-\mathrm{CH}-\mathrm{NH}, J 2.8 \mathrm{~Hz}), 6.38 \mathrm{q}(1 \mathrm{H}$, exo$\mathrm{CH}=\mathrm{CH}-\mathrm{CH}-\mathrm{CH}-\mathrm{NH}, J 3.0 \mathrm{~Hz}), 6.45 \mathrm{~d}(1 \mathrm{H}$, endo- $\mathrm{NH}, J$ 7.2 Hz), 7.11-7.15 m (1H, 5-H Ph), 7.22-7.27 m (1H, 6-H $\mathrm{Ph}), 7.74-7.78 \mathrm{~m}(1 \mathrm{H}, 2-\mathrm{H} \mathrm{Ph}), 8.42 \mathrm{~s}(1 \mathrm{H}$, endo-NH$\mathrm{Ph}), 8.50 \mathrm{~s}\left(1 \mathrm{H}\right.$, exo-NH-Ph). ${ }^{19} \mathrm{~F}$ (DMSO- $\left.d_{6}\right), \delta, \mathrm{ppm}$ : -126.38 . Found, \%: C 59.93; H 5.02; N 9.97; F 6.80. $\mathrm{C}_{14} \mathrm{H}_{14} \mathrm{ClFN}_{2} \mathrm{O}$. Calculated, \%: C 59.90; H 5.03; N 9.98; F 6.77. $M 280.73$.

1-(Bicyclo[2.2.1]hept-5-en-2-yl)-3-(3-chloro-2-fluorophenyl)urea (4k) was prepared similarly to compound $4 \mathrm{a}$ from $0.2 \mathrm{~g}(1.48 \mathrm{mmol})$ of bicyclo[2.2.1]hept-5-en-2-yl isocyanate (2), $0.214 \mathrm{~g}(1.48 \mathrm{mmol})$ of 3-chloro-2-fluoroaniline (3k), and $0.2 \mathrm{~mL}(1.48 \mathrm{mmol})$ of triethylamine. Yield $0.106 \mathrm{~g}(25 \%), \mathrm{mp} 201-202^{\circ} \mathrm{C}$. ${ }^{1} \mathrm{H}\left(\mathrm{DMSO}-d_{6}\right), \delta$, ppm: 0.62 d.t $\left(1 \mathrm{H}\right.$, endo- $\mathrm{C} \underline{\mathrm{H}}-\mathrm{CH}_{2}-$ $\left.\mathrm{CH}-\mathrm{NH}, J_{1} 11.9, J_{2} 3.2 \mathrm{~Hz}\right), 1.18$ d.t $\left(1 \mathrm{H}\right.$, exo- $\mathrm{CH}-\mathrm{CH}_{2}-$ $\left.\mathrm{CH}-\mathrm{NH}, J_{1} 12.0, J_{2} 3.4 \mathrm{~Hz}\right), 1.34 \mathrm{q}\left(2 \mathrm{H}\right.$, endo- $\mathrm{CH}_{2}, J$ 
$8.4 \mathrm{~Hz}), 1.44 \mathrm{q}\left(2 \mathrm{H}\right.$, exo- $\left.\mathrm{CH}_{2}, J 8.4 \mathrm{~Hz}\right), 2.13$ d.d.d $(1 \mathrm{H}$, $\left.\mathrm{C} \underline{\mathrm{H}}-\mathrm{CH}-\mathrm{NH}, J_{1} 12.2, J_{2} 8.8, J_{3} 3.8 \mathrm{~Hz}\right), 2.69 \mathrm{~s}(1 \mathrm{H}$, endo$\left.\mathrm{CH}-\mathrm{CH}_{2}-\mathrm{CH}-\mathrm{NH}\right), 2.81 \mathrm{~s}\left(1 \mathrm{H}\right.$, exo-CH- $\left.\underline{\mathrm{H}}_{2}-\mathrm{CH}-\mathrm{NH}\right)$, $2.85 \mathrm{~s}\left(1 \mathrm{H}\right.$, endo- $\left.\mathrm{CH}-\mathrm{CH}_{2}-\mathrm{CH}-\mathrm{NH}\right), 2.93 \mathrm{~s}(1 \mathrm{H}$, exo$\left.\mathrm{CH}-\mathrm{C}_{2}-\mathrm{CH}-\mathrm{NH}\right), 3.45-3.50 \mathrm{~m}(1 \mathrm{H}$, endo- $\mathrm{C} \underline{\mathrm{H}}-\mathrm{NH})$, 4.24 heptet $(1 \mathrm{H}$, exo-Cㅍ- $-\mathrm{NH}, J 3.7 \mathrm{~Hz}), 6.03 \mathrm{q}(1 \mathrm{H}$, endo- $\mathrm{CH}=\mathrm{CH}-\mathrm{CH}-\mathrm{CH}-\mathrm{NH}, J 3.3 \mathrm{~Hz}), 6.08 \mathrm{q}(1 \mathrm{H}$, exo- $\mathrm{CH}=\mathrm{CH}-\mathrm{CH}-\mathrm{CH}-\mathrm{NH}, J 3.1 \mathrm{~Hz}), 6.17 \mathrm{q}(1 \mathrm{H}$, endo$\mathrm{CH}=\mathrm{C} \underline{\mathrm{H}}-\mathrm{CH}-\mathrm{CH}-\mathrm{NH}, J 2.8 \mathrm{~Hz}), 6.33 \mathrm{~d}(1 \mathrm{H}$, exo-NH, $J 8.2 \mathrm{~Hz}), 6.39$ q $(1 \mathrm{H}$, exo-CH=C$\underline{\mathrm{H}}-\mathrm{CH}-\mathrm{CH}-\mathrm{NH}, J$ $3.0 \mathrm{~Hz}), 6.92 \mathrm{~d}(1 \mathrm{H}$, endo-NH, J 7.2 Hz), 7.03-7.12 m $(2 \mathrm{H}, 4,5-\mathrm{H} \mathrm{Ph}), 8.14$ q.d $\left(1 \mathrm{H}, 6-\mathrm{H} \mathrm{Ph}, J_{1} 7.8, J_{2} 2.1 \mathrm{~Hz}\right)$, $8.29 \mathrm{~s}\left(1 \mathrm{H}\right.$, endo-NH-Ph), $8.38 \mathrm{~s}\left(1 \mathrm{H}\right.$, exo-NH-Ph). ${ }^{19} \mathrm{~F}$ NMR spectrum (DMSO- $\left.d_{6}\right), \delta$, ppm: -133.47 . Mass spectrum, $m / z\left(I_{\text {rel }}, \%\right): 280(5.0)[M]^{+}, 214$ (41.0) [FCl-Ph$\left.\mathrm{NH}-\mathrm{C}(\mathrm{O})-\mathrm{NH}-\mathrm{CH}=\mathrm{CH}_{2}\right]^{+}, 171(12.5)[\mathrm{FCl}-\mathrm{Ph}-\mathrm{NCO}]^{+}$, 145 (100) [ $\left.\mathrm{FCl}-\mathrm{Ph}-\mathrm{NH}_{2}\right]^{+}$. Found, \%: C 59.89; H 4.99; $\mathrm{N}$ 10.01; F 6.79. $\mathrm{C}_{14} \mathrm{H}_{14} \mathrm{ClFN}_{2} \mathrm{O}$. Calculated, \%: C 59.90; H 5.03; N 9.98; F 6.77. $M 280.73$.

1-(Bicyclo[2.2.1] hept-5-en-2-yl)-3-(4-chloro-3-fluorophenyl)urea (4I) was prepared similarly to compound 4a from $0.2 \mathrm{~g}(1.48 \mathrm{mmol})$ of bicyclo[2.2.1]hept-5-en-2-yl isocyanate (2), $0.214 \mathrm{~g}(1.48 \mathrm{mmol})$ of 4-chloro-3-fluoroaniline (3l), and $0.2 \mathrm{~mL}(1.48 \mathrm{mmol})$ of triethylamine. Yield $0.27 \mathrm{~g}(65 \%), \mathrm{mp} 93-94^{\circ} \mathrm{C}$. ${ }^{1} \mathrm{H}$ NMR spectrum (DMSO- $\left.d_{6}\right), \delta$, ppm: 0.62 d.t $\left(1 \mathrm{H}\right.$, endo- $\underline{\mathrm{H}}-\mathrm{CH}_{2}-\mathrm{CH}-$ $\left.\mathrm{NH}, J_{1} 11.9, J_{2} 3.2 \mathrm{~Hz}\right), 1.20$ d.t $\left(1 \mathrm{H}\right.$, exo-C $\underline{\mathrm{H}}-\mathrm{CH}_{2}-$ $\left.\mathrm{CH}-\mathrm{NH}, J_{1} 12.0, J_{2} 3.4 \mathrm{~Hz}\right), 1.34 \mathrm{q}\left(2 \mathrm{H}\right.$, endo- $\mathrm{CH}_{2}, J$ $8.4 \mathrm{~Hz}), 1.56 \mathrm{q}\left(2 \mathrm{H}\right.$, exo- $\left.\mathrm{CH}_{2}, J 8.4 \mathrm{~Hz}\right), 2.12$ d.d.d $(1 \mathrm{H}$, $\left.\mathrm{C} \underline{\mathrm{H}}-\mathrm{CH}-\mathrm{NH}, J_{1} 12.2, J_{2} 8.8, J_{3} 3.8 \mathrm{~Hz}\right), 2.69 \mathrm{~s}(1 \mathrm{H}$, endo$\left.\mathrm{CH}-\mathrm{CH}_{2}-\mathrm{CH}-\mathrm{NH}\right), 2.81 \mathrm{~s}\left(1 \mathrm{H}\right.$, exo- $\left.\mathrm{CH}-\mathrm{CH}_{2}-\mathrm{CH}-\mathrm{NH}\right)$, $2.83 \mathrm{~s}\left(1 \mathrm{H}\right.$, endo- $\left.\mathrm{CH}-\mathrm{CH}_{2}-\mathrm{CH}-\mathrm{NH}\right), 2.93 \mathrm{~s}(1 \mathrm{H}$, exo$\left.\mathrm{CH}-\mathrm{C}_{2}-\mathrm{CH}-\mathrm{NH}\right), 3.45-3.50 \mathrm{~m}(1 \mathrm{H}$, endo- $\mathrm{C} \underline{\mathrm{H}}-\mathrm{NH})$, 4.24 heptet $(1 \mathrm{H}$, exo-C $\underline{\mathrm{H}}-\mathrm{NH}, J 3.7 \mathrm{~Hz}), 5.84 \mathrm{~d}(1 \mathrm{H}$, exo$\mathrm{NH}, J 8.2 \mathrm{~Hz}), 6.03 \mathrm{q}(1 \mathrm{H}$, endo- $\mathrm{C} \underline{\mathrm{H}}=\mathrm{CH}-\mathrm{CH}-\mathrm{CH}-\mathrm{NH}$, $J 3.3 \mathrm{~Hz}), 6.08$ q $(1 \mathrm{H}$, exo- $\underline{\mathrm{H}}=\mathrm{CH}-\mathrm{CH}-\mathrm{CH}-\mathrm{NH}, J$ $3.1 \mathrm{~Hz}), 6.17$ q $(1 \mathrm{H}$, endo- $\mathrm{CH}=\mathrm{C} \underline{\mathrm{H}}-\mathrm{CH}-\mathrm{CH}-\mathrm{NH}, J$ $2.8 \mathrm{~Hz}), 6.38 \mathrm{q}(1 \mathrm{H}$, exo- $\mathrm{CH}=\mathrm{C} \underline{\mathrm{H}}-\mathrm{CH}-\mathrm{CH}-\mathrm{NH}, J$ $3.0 \mathrm{~Hz}), 6.58 \mathrm{~d}(1 \mathrm{H}$, endo-NH, $J 7.2 \mathrm{~Hz}), 7.00 \mathrm{~d} . \mathrm{d}(1 \mathrm{H}$, 6-H Ph, $\left.J_{1} 8.5, J_{2} 2.1 \mathrm{~Hz}\right), 7.36 \mathrm{t}(1 \mathrm{H}, 5-\mathrm{H} \mathrm{Ph}, J 8.7 \mathrm{~Hz})$, 7.62 d.d $\left(1 \mathrm{H}, 6-\mathrm{H} \mathrm{Ph}, J_{1} 12.5, J_{2} 2.5 \mathrm{~Hz}\right), 8.69 \mathrm{~s}(1 \mathrm{H}$, endo-NH-Ph), $8.72 \mathrm{~s}\left(1 \mathrm{H}\right.$, exo-NH-Ph). ${ }^{19} \mathrm{~F}$ NMR spectrum (DMSO- $\left.d_{6}\right), \delta$, ppm: -115.34 . Found, \%: C 59.92; $\mathrm{H}$ 5.01; N 10.02; F 6.71. $\mathrm{C}_{14} \mathrm{H}_{14} \mathrm{ClFN}_{2} \mathrm{O}$. Calculated, \%: C 59.90; H 5.03; N 9.98; F 6.77. M 280.73.

1-(Bicyclo[2.2.1]hept-5-en-2-yl)-3-(4-chloro-2-fluorophenyl)urea $(\mathbf{4 m})$ was prepared similarly to compound $4 \mathbf{a}$ from $0.2 \mathrm{~g}(1.48 \mathrm{mmol})$ of bicyclo[2.2.1]- hept-5-en-2-yl isocyanate (2), $0.214 \mathrm{~g}(1.48 \mathrm{mmol})$ of 4-chloro-2-fluoroaniline (3m), and $0.2 \mathrm{~mL}(1.48 \mathrm{mmol})$ of triethylamine. Yield $0.17 \mathrm{~g}(41 \%), \mathrm{mp} 147-148^{\circ} \mathrm{C}$. ${ }^{1} \mathrm{H}$ NMR spectrum (DMSO- $\left.d_{6}\right), \delta$, ppm: 0.61 d.t $(1 \mathrm{H}$, endo- $\left.\mathrm{C}-\mathrm{CH}_{2}-\mathrm{CH}-\mathrm{NH}, J_{1} 11.9, J_{2} 3.2 \mathrm{~Hz}\right), 1.16$ d.t $(1 \mathrm{H}$, exo-C $\left.\mathrm{H}-\mathrm{CH}_{2}-\mathrm{CH}-\mathrm{NH}, J_{1} 12.0, J_{2} 3.4 \mathrm{~Hz}\right), 1.34 \mathrm{q}(2 \mathrm{H}$, endo- $\left.\mathrm{CH}_{2}, J 8.4 \mathrm{~Hz}\right), 1.44 \mathrm{q}\left(2 \mathrm{H}\right.$, exo- $\left.\mathrm{CH}_{2}, J 8.4 \mathrm{~Hz}\right)$, 2.12 d.d.d (1H, $\left.\underline{\mathrm{CH}}-\mathrm{CH}-\mathrm{NH}, J_{1} 12.2, J_{2} 8.8, J_{3} 3.8 \mathrm{~Hz}\right)$, $2.69 \mathrm{~s}\left(1 \mathrm{H}\right.$, endo- $\left.\mathrm{CH}-\mathrm{CH}_{2}-\mathrm{CH}-\mathrm{NH}\right), 2.81 \mathrm{~s}(1 \mathrm{H}$, exo$\left.\mathrm{CH}-\mathrm{C}_{2}-\mathrm{CH}-\mathrm{NH}\right), 2.84 \mathrm{~s}\left(1 \mathrm{H}\right.$, endo- $\mathrm{CH}-\mathrm{C}_{2}-\mathrm{CH}-$ $\mathrm{NH}), 2.93 \mathrm{~s}\left(1 \mathrm{H}\right.$, exo-CH- $\left.\underline{\mathrm{H}}_{2}-\mathrm{CH}-\mathrm{NH}\right), 3.47-3.52 \mathrm{~m}$ $(1 \mathrm{H}$, endo- $\underline{\mathrm{H}}-\mathrm{NH}), 4.24$ heptet $(1 \mathrm{H}$, exo-C$-\mathrm{HH}, J$ $3.7 \mathrm{~Hz}), 6.03$ q $(1 \mathrm{H}$, endo- $\mathrm{C} \underline{\mathrm{H}}=\mathrm{CH}-\mathrm{CH}-\mathrm{CH}-\mathrm{NH}, J$ $3.3 \mathrm{~Hz}), 6.08$ q $(1 \mathrm{H}$, exo $-\mathrm{CH}=\mathrm{CH}-\mathrm{CH}-\mathrm{CH}-\mathrm{NH}, J$ $3.1 \mathrm{~Hz}), 6.17 \mathrm{q}(1 \mathrm{H}$, endo- $\mathrm{CH}=\mathrm{C} \underline{\mathrm{H}}-\mathrm{CH}-\mathrm{CH}-\mathrm{NH}, J$ $2.8 \mathrm{~Hz}), 6.30 \mathrm{~d}(1 \mathrm{H}$, exo-NH, $J 8.2 \mathrm{~Hz}), 6.38 \mathrm{q}(1 \mathrm{H}$, exo$\mathrm{CH}=\mathrm{CH}-\mathrm{CH}-\mathrm{CH}-\mathrm{NH}, J 3.0 \mathrm{~Hz}), 6.89 \mathrm{~d}(1 \mathrm{H}$, endo- $\mathrm{NH}$, $J 7.2 \mathrm{~Hz}), 7.15 \mathrm{t}(1 \mathrm{H}, 5-\mathrm{H} \mathrm{Ph}, J 6.9 \mathrm{~Hz}), 7.37 \mathrm{t} . \mathrm{d}(1 \mathrm{H}$, 6-H Ph, $\left.J_{1} 11.4, J_{2} 2.4 \mathrm{~Hz}\right), 8.20 \mathrm{t}(1 \mathrm{H}, 3-\mathrm{H} \mathrm{Ph}, J 8.9 \mathrm{~Hz})$, $8.23 \mathrm{~d}(1 \mathrm{H}$, endo-NH-Ph, $J 2.3 \mathrm{~Hz}), 8.32 \mathrm{~d}(1 \mathrm{H}$, exo-NH$\mathrm{Ph}, J 2.3 \mathrm{~Hz}$ ). ${ }^{19} \mathrm{~F}$ NMR spectrum (DMSO- $d_{6}$ ), $\delta$, ppm: -128.05 . Mass spectrum, $m / z\left(I_{\mathrm{rel}}, \%\right): 280(3.0)[M]^{+}$, 14 (41.5) $\left[\mathrm{FCl}-\mathrm{Ph}-\mathrm{NH}-\mathrm{C}(\mathrm{O})-\mathrm{NH}-\mathrm{CH}=\mathrm{CH}_{2}\right]^{+}, 171$ (15.3) $[\mathrm{FCl}-\mathrm{Ph}-\mathrm{NCO}]^{+}, 145$ (100) [FCl-Ph-NH $]^{+}$. Found, \%: C 59.95; H 5.05; N 9.99; F 6.78. $\mathrm{C}_{14} \mathrm{H}_{14} \mathrm{ClFN}_{2} \mathrm{O}$. Calculated, \%: C 59.90; H 5.03; N 9.98; F 6.77. M 280.73.

1-(Bicyclo[2.2.1] hept-5-en-2-yl)-3-(5-chloro-2-fluorophenyl)urea (4n) was prepared similarly to compound 4a from $0.2 \mathrm{~g}(1.48 \mathrm{mmol})$ of bicyclo[2.2.1]hept-5-en-2-yl isocyanate (2), $0.214 \mathrm{~g}(1.48 \mathrm{mmol})$ of 5-chloro-2-fluoroaniline (3n), and $0.2 \mathrm{~mL}(1.48 \mathrm{mmol})$ of triethylamine. Yield $0.11 \mathrm{~g}(27 \%)$, mp $195-196^{\circ} \mathrm{C}$. ${ }^{1} \mathrm{H}$ NMR spectrum (DMSO- $\left.d_{6}\right), \delta$, ppm: 0.62 d.t $(1 \mathrm{H}$, endo- $\left.\mathrm{C}-\mathrm{H}-\mathrm{CH}_{2}-\mathrm{CH}-\mathrm{NH}, J_{1} 11.9, J_{2} 3.2 \mathrm{~Hz}\right), 1.17$ d.t $(1 \mathrm{H}$, exo- $\left.\mathrm{CH}-\mathrm{CH}_{2}-\mathrm{CH}-\mathrm{NH}, J_{1} 12.0, J_{2} 3.4 \mathrm{~Hz}\right), 1.34 \mathrm{q}(2 \mathrm{H}$, endo- $\left.\mathrm{CH}_{2}, J 8.4 \mathrm{~Hz}\right), 1.44 \mathrm{q}\left(2 \mathrm{H}\right.$, exo- $\left.\mathrm{CH}_{2}, J 8.4 \mathrm{~Hz}\right)$, 2.13 d.d.d ( $\left.1 \mathrm{H}, \mathrm{C} \underline{\mathrm{H}}-\mathrm{CH}-\mathrm{NH}, J_{1} 12.2, J_{2} 8.8, J_{3} 3.8 \mathrm{~Hz}\right)$, $2.69 \mathrm{~s}\left(1 \mathrm{H}\right.$, endo- $\left.\mathrm{CH}-\mathrm{CH}_{2}-\mathrm{CH}-\mathrm{NH}\right), 2.81 \mathrm{~s}(1 \mathrm{H}$, exo$\left.\mathrm{CH}-\mathrm{CH}_{2}-\mathrm{CH}-\mathrm{NH}\right), 2.85 \mathrm{~s}\left(1 \mathrm{H}\right.$, endo- $\mathrm{CH}-\mathrm{CH}_{2}-\mathrm{CH}-$ $\mathrm{NH}), 2.93 \mathrm{~s}\left(1 \mathrm{H}\right.$, exo-CH- $\left.\underline{\mathrm{H}}_{2}-\mathrm{CH}-\mathrm{NH}\right), 3.47-3.52 \mathrm{~m}$ $(1 \mathrm{H}$, endo- $\mathrm{C} \underline{\mathrm{H}}-\mathrm{NH}), 4.24$ heptet $(1 \mathrm{H}$, exo- $\mathrm{C} \underline{\mathrm{H}}-\mathrm{NH}, J$ $3.7 \mathrm{~Hz}), 6.03$ q $(1 \mathrm{H}$, endo- $\underline{\mathrm{H}}=\mathrm{CH}-\mathrm{CH}-\mathrm{CH}-\mathrm{NH}, J$ $3.3 \mathrm{~Hz}), 6.08 \mathrm{q}(1 \mathrm{H}$, exo $-\mathrm{C} \underline{\mathrm{H}}=\mathrm{CH}-\mathrm{CH}-\mathrm{CH}-\mathrm{NH}, J$ $3.1 \mathrm{~Hz}), 6.17 \mathrm{q}(1 \mathrm{H}$, endo- $\mathrm{CH}=\mathrm{C} \underline{\mathrm{H}}-\mathrm{CH}-\mathrm{CH}-\mathrm{NH}, J$ $2.8 \mathrm{~Hz}), 6.38 \mathrm{q}(1 \mathrm{H}$, exo- $\mathrm{CH}=\mathrm{C} \underline{\mathrm{H}}-\mathrm{CH}-\mathrm{CH}-\mathrm{NH}, J$ $3.0 \mathrm{~Hz}), 6.38 \mathrm{~d}(1 \mathrm{H}$, exo-NH, J8.2 Hz), 6.91-6.98 m $(1 \mathrm{H}$, $5-\mathrm{H} \mathrm{Ph}), 7.12 \mathrm{~d}(1 \mathrm{H}$, endo- $\mathrm{NH}, J 7.8 \mathrm{~Hz}), 7.22$ d.d $(1 \mathrm{H}$, $\left.6-\mathrm{H} \mathrm{Ph}, J_{1} 11.0, J_{2} 2.2 \mathrm{~Hz}\right), 8.27-8.31 \mathrm{~m}(1 \mathrm{H}, 2-\mathrm{H} \mathrm{Ph})$, 
$8.37 \mathrm{~d}(1 \mathrm{H}$, endo- $\mathrm{NH}-\mathrm{Ph}, J 2.6 \mathrm{~Hz}), 8.43 \mathrm{~d}(1 \mathrm{H}$, exo-NH$\mathrm{Ph}, J 2.6 \mathrm{~Hz}$ ). ${ }^{19} \mathrm{~F}$ NMR spectrum (DMSO- $\left.d_{6}\right), \delta$, ppm: -133.05 . Mass spectrum, $m / z\left(I_{\text {rel }}, \%\right): 280(4.0)[M]^{+}, 214$ (38.0) [FCl-Ph-NH-C(O)-NH-CH= $\left.\mathrm{CH}_{2}\right]^{+}, 171(14.0)$ [FCl-Ph-NCO] $]^{+}, 145(100)$ [FCl-Ph- $\left.\mathrm{NH}_{2}\right]^{+}$. Found, \%: C 59.88; $\mathrm{H} 4.98 ; \mathrm{N}$ 9.95; F 6.81. $\mathrm{C}_{14} \mathrm{H}_{14} \mathrm{ClFN}_{2} \mathrm{O}$. Calculated, \%: C 59.90; H 5.03; N 9.98; F 6.77. M 280.73.

\section{FUNDING}

The work was financially supported by the Russian Science Foundation (project no. 19-73-10002).

\section{CONFLICT OF INTEREST}

The authors declare no conflict of interest.

\section{REFERENCES}

1. Danilov, D.V., Burmistrov, V.V., and Butov, G.M., Russ. J. Org. Chem., 2020, vol. 56, p. 1132. https://doi.org/10.1134/S1070428020070027

2. Butov, G.M., Burmistrov, V.V., and Pitushkin, D.A., Russ. J. Org. Chem., 2017, vol. 53, p. 673. https://doi.org/10.1134/S1070428017050050

3. Kim, Y., George, D., Prior, A.M., Prasain, K., Hao, S., Le, D.D., Hua, D.H., and Chang, K.O., Eur. J. Med. Chem., 2012, vol. 50, p. 311.

10.1016/j.ejmech.2012.02.010

4. Park, S.J., Kim, Y.G., and Park, H.J., J. Am. Chem. Soc., 2011, vol. 133, p. 10094.

https://doi.org/10.1021/ja1098325

5. Mariani, R. and Maffioli, S.I., Curr. Med. Chem., 2009, vol. 16, p. 430. https://doi.org/10.2174/092986709787315559

6. Hwang, S.H., Wecksler, A.T., Zhang, G., Morisseau, C., Nguyen, L.V., Fu, S.H., and Hammock, B.D., Bioorg. Med. Chem. Lett., 2013, vol. 23, p. 3732.

https://doi.org/10.1016/j.bmcl.2013.05.011

7. Morisseau, C. and Hammock, B.D., Annu. Rev. Pharmacol. Toxicol., 2013, vol. 53, p. 37.

https://doi.org/10.1146/annurev-pharmtox-011112140244

8. Fleming, I., Rueben, A., Popp, R., Fisslthaler, B., Schrodt, S., Sander, A., Haendeler, J., Falck, J.R., Morisseau, C., Hammock, B.D., and Busse, R., Arterioscler. Thromb Vasc. Biol., 2007, vol. 27, p. 2612. https://doi.org/10.1161/ATVBAHA.107.152074
9. Codony, S., Valverde, E., Leiva, R., Brea, J., Loza, M.I., Morisseau, C., Hammock, B.D., and Vázquez, S., Bioorg. Med. Chem., 2019, vol. 27, p. 115078. https://doi.org/10.1016/j.bmc.2019.115078

10. Shen, H.C. and Hammock, B.D., J. Med. Chem., 2012, vol. 55 , p. 1789. https://doi.org/10.1021/jm201468j

11. Müller, F., Arch. Pharm., 1900, vol. 238, p. 366. https://doi.org/10.1002/ardp.19002380507

12. Aschan, O., Chem. Ber., 1907, vol. 40, p. 4918. https://doi.org/10.1002/cber.190704004160

13. Ivankin, A.V., Panferov, V.I., Fakhretdinov, Kh.A., Vostrikova, N.L., and Kulikovskii, A.V., Lesnoi Vestn., 2015, vol. 19, p. 46.

14. Wiseman, E.H., Pereira, J.N., Finger, K.F., and Pinson, R.Jr., J. Med. Chem., 1965, vol. 8, p. 777. https://doi.org/10.1021/jm00330a010

15. Iwakura, Y., Nakabayashi, N., and Lee, M.H., Macromol. Chem. Phys., 1964, vol. 78, p. 157. https://doi.org/10.1002/macp.1964.020780113

16. Diveley, W.R., Buntin, G.A., and Lohr, A.D., J. Org. Chem., 1969, vol. 34, p. 616. https://doi.org/10.1021/jo01255a029

17. Boehme, W.R., Graeme, M.L., Scharpf, W.G., Siegmund, E., Schipper, E., and Tobkes, M., J. Med. Chem., 1961, vol. 4, p. 183. https://doi.org/10.1021/jm50017a014

18. Takaya, T. and Tozuka, Z., US Patent no. 4349552, 1982.

19. Burmistrov, V.V., D’yachenko, V.S., Rasskazova, E.V., and Butov, G.M., Russ. J. Org. Chem., 2019, vol. 55, p. 1166. https://doi.org/10.1134/S1070428019080165

20. Burmistrov, V.V., Danilov, D.V., D’yachenko, V.S., Rasskazova, E.V., and Butov, G.M., Russ. J. Org. Chem., 2020, vol. 56, p. 735.

https://doi.org/10.1134/S1070428020050024

21. Lipinski, C.A., Lombardo, F., Dominy, B.W., and Feeney, P.J., Adv. Drug Del. Rev., 2001, vol. 46, p. 3. https://doi.org/10.1016/S0169-409X(00)00129-0

22. Becker, K., Salar-Behzadi, S., and Zimmer, A., Pharm. Res., 2015, vol. 32, p. 1519. https://doi.org/10.1007/s11095-015-1661-y

23. Burmistrov, V.V., Danilov, D.V., Rasskazova, E.V., and Butov, G.M., Russ. J. Org. Chem., 2020, vol. 56, p. 983 https://doi.org/10.1134/S1070428020060032

24. Kuznetsov, D.V., Burmistrov, V.V., and Butov, G.M., Izv. $\operatorname{Vog} G T U, 2018$, vol. 12, p. 7. 